\title{
Influence of Adjuvants on the Efficacy of Tolpyralate plus Atrazine for the Control of Annual Grass and Broadleaf Weeds in Corn with and without Roundup WeatherMAX ${ }^{\circledR}$
}

\author{
Nicole M. Langdon', Nader Soltani1 ${ }^{*}$, Alan J. Raedar', Darren E. Robinson', David C. Hooker1, \\ Peter H. Sikkema1
}

${ }^{1}$ University of Guelph Ridgetown Campus, Ridgetown, Canada

${ }^{2}$ ISK Biosciences Inc., Concord, OH, USA

Email: *soltanin@uoguelph.ca

How to cite this paper: Langdon, N.M., Soltani, N., Raedar, A.J., Robinson, D.E., Hooker, D.C. and Sikkema, P.H. (2020) Influence of Adjuvants on the Efficacy of Tolpyralate plus Atrazine for the Control of Annual Grass and Broadleaf Weeds in Corn with and without Roundup WeatherMAX. American Journal of Plant Sciences, 11, 465-495.

https://doi.org/10.4236/ajps.2020.113034

Received: February 9, 2020

Accepted: March 24, 2020

Published: March 27, 2020

Copyright $\odot 2020$ by author(s) and Scientific Research Publishing Inc. This work is licensed under the Creative Commons Attribution International License (CC BY 4.0).

http://creativecommons.org/licenses/by/4.0/

\begin{abstract}
Tolpyralate is a new HPPD-inhibiting herbicide that is efficacious on annual grass and broadleaf weed species in corn. For maximum herbicide performance of tolpyralate, it is recommended that atrazine is tank mixed with tolpyralate along with the adjuvants methylated seed oil concentrate (MSO) plus urea ammonia nitrate (UAN). A common use pattern of tolpyralate plus atrazine will be in a tank mix with Roundup WeatherMAX ${ }^{\oplus}$ due to the high proportion of corn acres that are seeded to Roundup Ready ${ }^{\oplus}$ hybrids in Eastern Canada. There is no information in the peer-reviewed literature if the adjuvant system in Roundup WeatherMAX ${ }^{\bullet}$ is adequate for optimal herbicide performance of tolpyralate plus atrazine, or if MSO and UAN are still required. Six field trials were conducted over two years near Ridgetown and Exeter, ON, Canada to determine if adjuvants are still required when tolpyralate plus atrazine is tank mixed with Roundup WeatherMAX ${ }^{\oplus}$ in corn. Tolpyralate plus atrazine plus MSO and Roundup WeatherMAX ${ }^{\oplus}$ plus tolpyralate plus atrazine provided excellent control of velvetleaf, pigweed spp, common ragweed, lambsquarters, ladysthumb, wild mustard, flower-of-an-hour, barnyardgrass and green foxtail in this study. Results of this study show that in the absence of Roundup WeatherMAX, weed control with tolpyralate plus atrazine was improved substantially with the addition of MSO; however, there was little to no increase in weed control with the addition of UAN. When tolpyralate plus atrazine was co-applied with Roundup WeatherMAX ${ }^{\oplus}$, there was no improvement in weed control with the addition of MSO and/or UAN.
\end{abstract}




\section{Keywords}

Biomass, Broadleaf Weeds, Corn, Efficacy, Grassweeds, Roundup WeatherMAX, Methylated Seed Oil, Urea Ammonia Nitrate, Weed Control, Yield, Zea mays L

\section{Introduction}

Weed interference during the early stages of corn development can cause physiological changes in the plant and lead to yield loss [1]. A study conducted across the corn producing regions of the United States and Canada found that in the absence of any weed management tactics, corn yield loss due to weed interference was 50\% [2]. The critical weed free period (CWFP) in corn delineates the time period during which weed interference must be minimized to prevent corn yield loss. The CWFP is crop specific due to the differences in crop growth and development and can vary across location and years [3]. Hall et al. 1992 [4] determined that the CWFP in Ontario for corn is from the 3 to 14 leaf-tip stage. Using the CWFP as a guideline to time POST herbicide applications can minimize corn yield and economic losses [3].

Tolpyralate is a new 4-hydroxyphenyl-pyruvate dioxygenase (HPPD)-inhibitor belonging to the benzoylpyrazole family. This is the second benzoylpyrazole herbicide registered for use in corn. Classified as a Group 27 herbicide, HPPD-inhibitors affect sensitive weeds by interrupting the biosynthesis of plastoquinone and tocopherol and stopping the synthesis of carotenoid pigments [5]. The inability of plants to quench singlet oxygen and other reactive oxygen species leads to light induced destruction of proteins, lipids and the photosynthetic complex, releasing free chlorophyll [5] [6]. Free chlorophyll generates additional singlet oxygen that causes destruction of leaf pigments and results in the characteristic white bleaching symptoms associated with HPPD-inhibiting herbicides [5] [6]. Tolpyralate (30 - $40 \mathrm{~g} \cdot \mathrm{a} \cdot \mathrm{ha}^{-1}$ ) is recommended to be co-applied with a photosystem II inhibiting herbicide, such as atrazine (560 - $1120 \mathrm{~g} \cdot \mathrm{a} \cdot \mathrm{ha}^{-1}$ ) [7]. Previous research by Metzger et al. 2018 [8] reported that the addition of atrazine to tolpyralate increases the speed of herbicide activity and expands the spectrum of weed species controlled. Among weed species, there is variability in sensitivity to tolpyralate. Weed species such as velvetleaf (Abutilon theophrasti Medik.), common ragweed (Ambrosia artemisiifolia L.), lambsquarters (Chenopodium album L.), redroot pigweed (Amaranthus retroflexus L.) and green foxtail (Setaria viridis (L.) P. Beauv.) are very sensitive to tolpyralate and were controlled $>90 \%$ with lower than label rates of tolpyralate $\left(15.5 \mathrm{~g} \cdot a \mathrm{i} \cdot \mathrm{ha}^{-1}\right)$; in contrast, ladysthumb (Persicaria maculosa Gray), wild mustard (Sinapis arvensis L.) and barnyardgrass (Echinochloa crus-galli (L.) P. Beauv.) required the addition of atrazine for $\geq 90 \%$ control [8]. Tolpyralate plus atrazine provides control of many of the common annual grass and broadleaf weeds in Ontario with an ade- 
quate margin of crop safety [9] [10].

The efficacy of tolypyrate on weeds may be improved with the addition of adjuvants, such as methylated seed oil (MSO) concentrate ( $1 \% \mathrm{v} / \mathrm{v})$ and a nitrogen source such as urea ammonium nitrate (UAN) or ammonium sulfate (AMS) $(2.5 \% \mathrm{v} / \mathrm{v})[11]$. Adjuvants are additives to a spray solution, or present in an herbicide formulation, that can enhance herbicidal activity or application characteristics [12]. There are various types of adjuvants that are grouped based on function; special purpose adjuvants indirectly influence spray solution characteristics, while activator adjuvants modify physical and chemical properties of an herbicide [13]. Activator adjuvants can decrease surface tension and contact angle of the spray droplet (angle between the leaf surface and the spray droplet), as well as influence spreading of the herbicide on the leaf surface [14]. MSO and UAN are activator adjuvants that are added to a spray mixture. Zhang et al. 2013 [15] reported that MSO increased herbicide retention, absorption and translocation in plant leaves. Gronwald et al. 1993 [16] reported an increase in herbicide absorption into the plant with the addition of a nitrogen fertilizer due to the influence of ammonium ions, further increasing herbicidal activity. Commonly, herbicides require the addition of an adjuvant for optimal performance. For example, topramezone absorption and translocation into the plant is enhanced with the addition of MSO [15]. Wozinac et al. 2003 [17] reported enhanced quinclorac efficacy when it was co-applied with MSO and a nitrogen fertilizer. In some cases, increased corn injury may occur with the addition of adjuvants such as MSO and UAN to tolpyralate, however plants rapidly recovered [9] [18].

Herbicide formulations may be sold preformulated with adjuvants. All glyphosate products sold in Canada are formulated with adjuvants; for example, Roundup WeatherMAX ${ }^{ø}$ contains proprietary surfactants for effective retention and absorption of the herbicide in the plant tissue [19]. Previous research has found that tank mixes of glyphosate formulations with additional herbicides may influence crop injury [20], weed control efficacy [21] [22] or herbicidal activity of the tank mix partners [23].

Adjuvants may have some benefits; however, they are an additional weed management cost. Across Ontario, adjuvants can cost up to $\$ 10.00 \mathrm{ha}^{-1}$ (Agris Co-operative, personal communication), in addition, they require the handling of multiple product containers thereby increasing time to fill sprayers in the field. In the literature, it is evident that there is an interaction between glyphosate and various herbicides, potentially a result of the aggressive adjuvant system in glyphosate products. Ninety-six percent of corn acres in Eastern Canada are seeded to Roundup Ready ${ }^{\circledR}$ hybrids (M. Reidy, Stratus, personal communication); therefore, tolpyralate plus atrazine will commonly be used in a tank mixture with glyphosate. The proprietary blend of surfactants in Roundup WeatherMAX ${ }^{\circledast}$ enhances the ability of the herbicide to move into the plant [19]; however, it has not been reported if the adjuvant system in Roundup WeatherMAX $^{\oplus}$ is adequate for optimal tolpyralate plus atrazine performance, or if additional adjuvants are still necessary. Therefore, the objective of this study was to 
determine if the adjuvants recommended on the tolpyralate label (MSO and UAN) are still required when Roundup WeatherMAX ${ }^{\circledR}$ is tank mixed with tolpyralate plus atrazine for the control of common annual weed species in Ontario.

\section{Materials and Methods}

Over a period of two years $(2018,2019)$, six field experiments were conducted at research sites near Ridgetown $\left(42.43956^{\circ} \mathrm{N},-81.88657^{\circ} \mathrm{E}\right)$ and Exeter $\left(50.71984^{\circ} \mathrm{N}\right.$, $-3.53019^{\circ} \mathrm{E}$ ), Ontario, Canada. Sites were managed with a three-crop rotation consisting of corn (Zea mays L.)-soybean (Glycine max (L.) Merr.)-winter wheat (Triticum aestivum L.) at Ridgetown and a five-crop rotation of corn-soybean -oat (Avena sativa)-dry bean (Phaseolus vulgaris L.)-winter wheat at Exeter. Seedbed preparation consisted of fall moldboard plowing followed by two passes in the spring with a field cultivator with rolling basket harrows. Fertilizer was applied to sites prior to planting, based on soil tests and nutritional needs of the crop. Table 1 lists location, year, soil type, $\mathrm{OM}$ and $\mathrm{pH}$, and planting, spray and harvest dates.

Corn hybrids were selected based on geographic suitability and seeded at approximately 83,500 seeds $\cdot \mathrm{ha}^{-1}$. Plots were $3 \mathrm{~m}$ wide (4 corn rows spaced $75 \mathrm{~cm}$ apart) and 8 or $10 \mathrm{~m}$ long at Ridgetown and Exeter, respectively. Experiments were designed as a two-way factorial: Factor One was Roundup WeatherMAX, and Factor Two was five tolpyralate plus atrazine treatments. Experiments were arranged in the field as a randomized complete block with 4 replications.

Herbicides were applied when the weeds were approximately $10 \mathrm{~cm}$ in height or diameter. Herbicides were applied with a $\mathrm{CO}_{2}$ pressurized, small-plot sprayer calibrated to deliver $200 \mathrm{~L} \cdot \mathrm{ha}^{-1}$ at $257 \mathrm{kPa}$. The $1.5 \mathrm{~m}$ spray boom was equipped with four ULD12002 nozzles (Pentair, New Brighton, MN, USA) spaced $50 \mathrm{~cm}$ apart producing a spray width of $2 \mathrm{~m}$. Factor One included Roundup Weather$\mathrm{MAX}^{\circledast}$ applied at 0 or $900 \mathrm{~g} \cdot \mathrm{ae} \cdot \mathrm{ha}^{-1}$. Factor Two treatments included: no tank mix partner, tolpyralate + atrazine $\left(30+560 \mathrm{~g} \cdot \mathrm{ai} \cdot \mathrm{ha}^{-1}\right)$, tolpyralate + atrazine +

Table 1. Soil characteristics, planting, spray and harvest dates for six trials near Ridgetown and Exeter, Ontario, Canada in 2018 and 2019 on control of common annual weeds with Roundup WeatherMAX ${ }^{\circledast}$ plus tolpyralate plus atrazine plus adjuvants, applied postemergence in corn.

\begin{tabular}{|c|c|c|c|c|c|c|c|}
\hline \multicolumn{8}{|c|}{ Soil Characteristics } \\
\hline Location & Year & Type & $\mathrm{OM}^{\mathrm{a}}(\%)$ & $\mathrm{pH}$ & Planting Date & Spray Date & Harvest Date \\
\hline \multirow[t]{4}{*}{ Ridgetown } & 2018 & Clay loam & 4.5 & 6.3 & May 25 & June 21 & Oct. 29 \\
\hline & 2018 & Clay loam & 4.0 & 6.9 & May 9 & June 6 & Nov. 8 \\
\hline & 2019 & Sandy clay loam & 5.4 & 6.6 & June 7 & July 2 & Oct. 28 \\
\hline & 2019 & Clay loam & 4.0 & 7.1 & June 9 & July 4 & Nov. 4 \\
\hline \multirow[t]{2}{*}{ Exeter } & 2018 & Clay loam & 4.5 & 7.8 & May 14 & June 7 & Oct. 20 \\
\hline & 2019 & Loam & 3.9 & 7.8 & June 18 & July 5 & $\mathrm{n} / \mathrm{a}^{\mathrm{b}}$ \\
\hline
\end{tabular}

${ }^{\mathrm{a}}$ Abbreviation: OM, organic matter. ${ }^{\mathrm{b}}$ Not harvested in 2019. 
MSO, tolpyralate + atrazine + UAN, and tolpyralate + atrazine + MSO + UAN. Methylated seed oil (MSO Concentrate ${ }^{\bowtie}$, Loveland Products Inc., Loveland CO) and $28 \%$ UAN were added at $0.5 \%$ and $2.5 \% \mathrm{v} / \mathrm{v}$, respectively.

Crop injury was evaluated at 1,2 and 4 weeks after application (WAA) and weed control was assessed at 2, 4 and 8 WAA. Visible crop injury and weed control were assessed on a percent scale, where 0 denotes no crop injury or weed control and 100 is complete plant death. Following the weed control assessment at 8 WAA, weed density and dry weight were determined by species by counting and recording the number of each species within two, $0.5 \mathrm{~m}^{2}$ quadrants per plot that were randomly placed between the center two rows of corn. After counting the number of weeds by species, they were cut at the soil surface, placed into brown paper bags by weed species, placed in a drier $(60 \mathrm{C})$ and the dry weight was recorded. Corn yield was determined at maturity by combining the two center rows in each plot; grain moisture content and weight were recorded. Grain yields were adjusted to $15.5 \%$ moisture for analysis and expressed as tonnes $\mathrm{ha}^{-1}$.

\section{Statistical Analysis}

Data were analyzed using SAS v 9.4 (SAS Institute, Cary, NC) and the GLIMMIX procedure. Data were pooled across years and location for analysis; normality assumptions were evaluated using PROC UNIVARIATE. Each location-year represented a site for a total of six sites in this study. When weed species were not present at a site, that site was excluded from analyses. Fixed effects were Factors One and Two and their interactions; significance was determined using F-tests. Site (location-year), replication within site and the interaction of the site with each fixed effect were designated as random effects and tested using Z-tests. For all analyses, significance was set to $\alpha=0.05$. The Shapiro-Wilk test of normality in addition to normality and scatter plots of studentized residuals were used to determine if residuals met the assumptions (homogeneous, had a mean of zero and were normally distributed). The appropriate distribution and link for each parameter that best met assumptions was used. Weed control data was transformed using arcsine square root, except wild mustard control at 2 WAA and flower-of-an-hour (Hibiscus trionum L.) control at 4 WAA that was assigned a normal distribution. Weed density and biomass were analyzed using a lognormal distribution. Yield was analyzed using a normal distribution. Treatment comparisons for main effects were performed only when the interaction was not significant. When the interaction was significant, simple effects were analyzed. Treatment comparisons were made based on least-square means using Tukey-Kramer's multiple range test and letter codes were assigned to illustrate statistically significant differences. Least-square means of each parameter were back-transformed to the original scale for presentation of results.

\section{Results and Discussion}

There were nine naturally-occurring weed species evaluated in this study. The 
broadleaf weed species were velvetleaf ( 4 sites, average density 4 plants. $\mathrm{m}^{-2}$ ), pigweed species (Amaranthus spp.) [AMASS] (5 sites, average density 17 plants $\left.\cdot \mathrm{m}^{-2}\right)$, common ragweed (6 sites, average density 35 plants $\mathrm{m}^{-2}$ ), lambsquarters ( 6 sites, average density 31 plants $\mathrm{m}^{-2}$ ), ladysthumb (5 sites, average density 4 plants $\cdot \mathrm{m}^{-2}$ ), wild mustard ( 2 sites, average density 143 plants $\cdot \mathrm{m}^{-2}$ ), and flower-of-an-hour ( 2 sites, average density 15 plants $\mathrm{m}^{-2}$ ). Pigweed species were grouped due to the presence of a mixed population of green pigweed (Amaranthus powellii (S.) Watson) and redroot pigweed (Amaranthus retroflexus L.), hybridization between these two species can occur (Weaver 2009). Of those species, lambsquarters, pigweed and common ragweed are ranked among the top 10 problem weed species in Ontario from a survey of farmers conducted by Bilyea 2016 [24]. The grass species present in this study were barnyardgrass ( 6 sites, average density 20 plants $\cdot \mathrm{m}^{-2}$ ) and green foxtail ( 6 sites, average density 70 plants $\left.\cdot \mathrm{m}^{-2}\right)$.

\subsection{Velvetleaf}

Analysis of variance determined that the interaction between Roundup WeatherMAX ${ }^{\oplus}$ and tolpyralate + atrazine was not significant for velvetleaf density $(P$ $=0.3347$ ) (Table 2). Averaged over all tolpyralate plus adjuvant combinations, the addition of Roundup WeatherMAX ${ }^{\circledR}$ reduced velvetleaf density $75 \%$ (Table 2). Averaged across 0 and $900 \mathrm{~g} \cdot \mathrm{ae} \cdot \mathrm{ha}^{-1}$ of Roundup WeatherMAX ${ }^{\oplus}$, tolpyralate + atrazine did not reduce velvetleaf density; the addition of either MSO or MSO + UAN to tolpyralate + atrazine reduced velvetleaf density up to $51 \%$. Velvetleaf control generally increased with the presence of Roundup WeatherMAX ${ }^{\circledR}$ and certain adjuvants, particularly MSO, producing a Roundup WeatherMAX ${ }^{\bullet}$ by tolpyralate + atrazine plus adjuvant interaction for weed control at 2, 4 and 8 WAA $(P<0.0001)$ and velvetleaf biomass $(P=0.0010)$ (Table 2$)$.

Tolpyralate + atrazine, in the absence of an adjuvant, controlled velvetleaf 49 , 42 and $42 \%$ at 2,4 or 8 WAA, respectively (Table 3 ). There was no improvement in velvetleaf control with the addition of UAN to tolpyralate + atrazine. Tolpyralate + atrazine + MSO controlled velvetleaf $94 \%, 92 \%$ and $86 \%$ at 2,4 and 8 WAA, respectively. There was no further increase in velvetleaf control when UAN was added to tolpyralate + atrazine + MSO. Similarly, there was no decrease in velvetleaf biomass with tolpyralate + atrazine or tolpyralate + atrazine + UAN. In contrast, tolpyralate + atrazine + MSO and tolpyralate + atrazine + MSO + UAN reduced velvetleaf biomass $95 \%$. The results from this study are consistent with Metzger et al. 2019 [10], who reported that tolpyralate + atrazine + MSO + UAN $\left(30+1000\right.$ g.ai $\left.\cdot \mathrm{ha}^{-1}\right)$, applied postemergence, controlled velvetleaf $92 \%-97 \%$ at 2 and 4 WAA and $72 \%$ at 8 WAA. Although no previous research evaluated the influence of MSO on tolpyralate + atrazine, Zhang et al. 2013 [15] reported that MSO enhanced the efficacy of topramezone, a Group 27 herbicide, on velvetleaf by 1.0 -fold due to increased absorption and translocation. Other research reported that bentazon absorption in velvetleaf was increased with the addition of a crop oil concentrate (COC) compared to UAN; 
Table 2. Significance of main effects and interactions for velvetleaf (Abutilon theophrasti Medik.) control, density and biomass with Roundup WeatherMAX ${ }^{\circledast}$ plus tolpyralate plus atrazine from four field experiments conducted in Ontario in 2018 and 2019.

\begin{tabular}{|c|c|c|c|c|c|}
\hline \multirow{2}{*}{ Main effects } & \multicolumn{3}{|c|}{ Visible Weed Control (\%) } & \multirow{2}{*}{$\begin{array}{l}\text { Density }{ }^{\mathrm{f}} \\
\left(\text { no } \cdot \mathrm{m}^{-2}\right)\end{array}$} & \multirow{2}{*}{$\begin{array}{r}\text { Biomass } \\
\left(\mathrm{g} \cdot \mathrm{m}^{-2}\right)\end{array}$} \\
\hline & $2 \mathrm{WAA}^{\mathrm{a}}$ & 4 WAA & 8 WAA & & \\
\hline \multicolumn{6}{|l|}{ Roundup WeatherMAX $X^{\oplus}\left(g \cdot a e \cdot h a^{-1}\right)$} \\
\hline 0 & 57 & 51 & 46 & $56.6 \mathrm{a}$ & 55.5 \\
\hline 900 & 97 & 95 & 92 & $14.3 \mathrm{~b}$ & 1.1 \\
\hline $\mathrm{SE}^{\mathrm{b}}$ & 3 & 3 & 2 & 2.8 & 4.1 \\
\hline Roundup WeatherMAX ${ }^{\circledast}$ P-value & 0.0004 & 0.0022 & $<0.0001$ & 0.0301 & 0.0116 \\
\hline \multicolumn{6}{|l|}{ Tolpyralate + atrazine treatments ${ }^{\mathcal{c}}$} \\
\hline No tank mix partner & 40 & 38 & 33 & $46.8 \mathrm{a}$ & 71.9 \\
\hline Tolpyralate + Atrazine & 78 & 74 & 70 & $29.5 \mathrm{ab}$ & 35.3 \\
\hline Tolpyralate + Atrazine $+\mathrm{MSO}^{\mathrm{d}}$ & 96 & 94 & 90 & $27.3 b$ & 2.8 \\
\hline Tolpyralate + Atrazine $+\mathrm{UAN}^{\mathrm{e}}$ & 80 & 73 & 70 & $28.5 \mathrm{ab}$ & 24.1 \\
\hline Tolpyralate + Atrazine $+\mathrm{MSO}+\mathrm{UAN}$ & 96 & 95 & 90 & $22.5 b$ & 2.3 \\
\hline $\mathrm{SE}^{\mathrm{b}}$ & 3 & 3 & 2 & 2.8 & 4.1 \\
\hline Tolpyralate + atrazine P-value & $<0.0001$ & $<0.0001$ & $<0.0001$ & 0.0056 & 0.0003 \\
\hline \multicolumn{6}{|l|}{ Interaction } \\
\hline $\begin{array}{c}\text { Roundup WeatherMAX }{ }^{\otimes \star} \text { tolpyralate }+ \\
\text { atrazine P-value }\end{array}$ & $<0.0001$ & $<0.0001$ & $<0.0001$ & 0.3347 & 0.0010 \\
\hline
\end{tabular}

${ }^{\mathrm{a}} \mathrm{WAA}$, weeks after treatment application. ${ }^{\mathrm{b}}$ Standard error of the mean. ${ }^{\mathrm{C}}$ Tolpyralate applied at $30 \mathrm{~g} \cdot \mathrm{ai} \cdot \mathrm{ha}^{-1}$; atrazine applied at $560 \mathrm{~g} \cdot \mathrm{ai} \cdot \mathrm{ha}^{-1} .{ }^{\mathrm{d}} \mathrm{MSO}, 0.50 \% \mathrm{v} / \mathrm{v}$. ${ }^{\mathrm{e} U A N}, 2.50 \% \mathrm{v} / \mathrm{v}$. ${ }^{\mathrm{f}}$ Density and biomass collected 8 WAA. Means within column followed by the same lowercase letter are not statistically different according to Tukey-Kramer's LSD $(P=0.05)$.

however, the addition of a COC or UAN doubled bentazon absorption in the leaves compared to when no adjuvant was used [25] [26]. In this study there was no improvement in velvetleaf control from the addition of UAN when MSO was present. Roundup WeatherMAX ${ }^{\circledR}$ controlled velvetleaf $88 \%-96 \%$ at 2, 4 and 8 WAA after application, and reduced velvetleaf biomass $98 \%$. There was no improvement in velvetleaf control from the addition of tolpyralate + atrazine with or without MSO, UAN or MSO + UAN. The addition of Roundup Weather$\mathrm{MAX}^{\oplus}$ to all tolpyralate + atrazine treatments increased velvetleaf control at 8 WAA and decreased velvetleaf biomass. However, no increase in control was observed from the addition of Roundup WeatherMAX ${ }^{\otimes}$ to treatments containing MSO at 2 WAA or MSO + UAN at 4 WAA. Starke and Oliver 1998 [23] found that tank mixtures of Roundup WeatherMAX ${ }^{\oplus}$ plus fomesafen, chlorimuron or sulfentrazone were antagonistic for velvetleaf control, however results of this study concluded that velvetleaf control is improved when Roundup Weather$\mathrm{MAX}^{\oplus}$ is added to tolpyralate + atrazine.

\subsection{Pigweed Species}

Data analysis determined that there was a statistically significant Roundup 
Table 3. Velvetleaf (Abutilon theophrasti Medik.) control 2, 4, and 8 WAA and biomass in corn with Roundup WeatherMAX ${ }^{\circledast}$ plus tolpyralate plus atrazine from four field experiments conducted in Ontario in 2018 and 2019.

\begin{tabular}{|c|c|c|c|}
\hline & \multicolumn{3}{|c|}{ Roundup WeatherMAX ${ }^{\infty}\left(\mathrm{g} \cdot \mathrm{ae} \cdot \mathrm{ha}^{-1}\right)$} \\
\hline & 0 & 900 & SE \\
\hline \multicolumn{4}{|l|}{ Visible Weed Control 2 WAA $A^{a}(\%)$} \\
\hline No tank mix partner & $0 c \mathrm{cY}$ & $96 a Z$ & 9 \\
\hline Tolpyralate + Atrazine $^{\mathrm{b}}$ & $49 \mathrm{bY}$ & $97 a Z$ & 5 \\
\hline Tolpyralate + Atrazine $+\mathrm{MSO}^{c}$ & $94 \mathrm{aZ}$ & $97 a Z$ & 0.7 \\
\hline Tolpyralate + Atrazine + UAN $^{\mathrm{d}}$ & $57 \mathrm{bY}$ & $96 a Z$ & 4 \\
\hline Tolpyralate + Atrazine $+\mathrm{MSO}+\mathrm{UAN}$ & $95 \mathrm{aZ}$ & $98 \mathrm{aZ}$ & 0.7 \\
\hline $\mathrm{SE}^{\mathrm{e}}$ & 4 & 0.3 & \\
\hline \multicolumn{4}{|l|}{ Visible Weed Control 4 WAA (\%) } \\
\hline No tank mix partner & $0 \mathrm{cY}$ & $93 \mathrm{aZ}$ & 8 \\
\hline Tolpyralate + Atrazine & $42 \mathrm{bY}$ & $96 a Z$ & 6 \\
\hline Tolpyralate + Atrazine + MSO & $92 \mathrm{aY}$ & $96 a Z$ & 1 \\
\hline Tolpyralate + Atrazine + UAN & $42 \mathrm{bY}$ & $96 a Z$ & 6 \\
\hline Tolpyralate + Atrazine $+\mathrm{MSO}+\mathrm{UAN}$ & $93 a Z$ & $96 a Z$ & 0.8 \\
\hline SE & 4 & 0.5 & \\
\hline \multicolumn{4}{|l|}{ Visible Weed Control 8 WAA (\%) } \\
\hline No tank mix partner & $0 \mathrm{cY}$ & $88 \mathrm{aZ}$ & 8 \\
\hline Tolpyralate + Atrazine & $42 \mathrm{bY}$ & $91 \mathrm{aZ}$ & 5 \\
\hline Tolpyralate + Atrazine + MSO & $86 a Y$ & $94 \mathrm{aZ}$ & 1 \\
\hline Tolpyralate + Atrazine + UAN & $44 \mathrm{bY}$ & $92 \mathrm{aZ}$ & 5 \\
\hline Tolpyralate + Atrazine + MSO + UAN & $84 \mathrm{aY}$ & $95 \mathrm{aZ}$ & 2 \\
\hline SE & 4 & 0.9 & \\
\hline \multicolumn{4}{|l|}{ Biomass $\left(\mathrm{g} \cdot \mathrm{m}^{-2}\right)$} \\
\hline No tank mix partner & $116.3 \mathrm{aY}$ & $2.1 \mathrm{aZ}$ & 16.5 \\
\hline Tolpyralate + Atrazine & $83.9 \mathrm{aY}$ & $0.6 \mathrm{aZ}$ & 7.43 \\
\hline Tolpyralate + Atrazine + MSO & $5.3 \mathrm{bZ}$ & $0.9 \mathrm{aZ}$ & 0.89 \\
\hline Tolpyralate + Atrazine + UAN & $73.9 \mathrm{aY}$ & $1.1 \mathrm{aZ}$ & 7.1 \\
\hline Tolpyralate + Atrazine + MSO + UAN & $4.4 \mathrm{bZ}$ & $0.8 \mathrm{aZ}$ & 0.75 \\
\hline SE & 8 & 0.3 & \\
\hline
\end{tabular}

${ }^{\mathrm{a}} \mathrm{WAA}$, weeks after treatment application. ${ }^{\mathrm{b}}$ Tolpyralate applied at $30 \mathrm{~g} \cdot \mathrm{ai} \cdot \mathrm{ha}^{-1}$; atrazine applied at 560 g.ai $\cdot \mathrm{ha}^{-1} \cdot{ }^{\mathrm{c}} \mathrm{MSO}, 0.50 \% \mathrm{v} / \mathrm{v} .{ }^{\mathrm{d}} \mathrm{UAN}, 2.50 \% \mathrm{v} / \mathrm{v}$. ${ }^{\mathrm{e} S t a n d a r d}$ error of the mean. Means within column followed by the same lowercase letter, or means within row followed by the same uppercase letter, are not statistically different according to Tukey-Kramer's LSD $(P=0.05)$.

WeatherMAX ${ }^{\circledR}$ by tolpyralate + atrazine interaction for pigweed control 2,4 , and 8 WAA $(P<0.001)$, density $(P=0.0001)$, and biomass $(P=0.0003)$ (Table 4$)$.

At 2, 4 and 8 WAA, tolpyralate + atrazine and tolpyralate + atrazine + UAN controlled pigweed $\leq 80 \%$ (Table 5 ). The addition of MSO or MSO + UAN to tolpyralate + atrazine increased pigweed control to $\geq 97 \%$. There was no increase 
Table 4. Significance of main effects and interactions for pigweed (Amaranthus spp. [AMASS]) control, density and biomass in corn with Roundup WeatherMAX ${ }^{\circledast}$ plus tolpyralate plus atrazine from five field experiments conducted in Ontario in 2018 and 2019.

\begin{tabular}{|c|c|c|c|c|c|}
\hline \multirow{2}{*}{ Main effects } & \multicolumn{3}{|c|}{ Visible Weed Control (\%) } & \multirow{2}{*}{$\begin{array}{l}\text { Density }{ }^{\mathrm{f}} \\
\left(\text { no }^{-m^{-2}}\right)\end{array}$} & \multirow{2}{*}{$\begin{array}{r}\text { Biomass } \\
\left(\mathrm{g} \cdot \mathrm{m}^{-2}\right)\end{array}$} \\
\hline & $2 \mathrm{WAA}^{\mathrm{a}}$ & $4 \mathrm{WAA}$ & $8 \mathrm{WAA}$ & & \\
\hline \multicolumn{6}{|l|}{ Roundup WeatherMAX $X^{\oplus}\left(g \cdot a e h a^{-1}\right)$} \\
\hline 0 & 71 & 72 & 70 & 2.5 & 5.04 \\
\hline 900 & 98 & 99 & 98 & 0.2 & 0.06 \\
\hline $\mathrm{SE}^{\mathrm{b}}$ & 2 & 3 & 2 & 0.4 & 2.47 \\
\hline Roundup WeatherMAX ${ }^{\oplus} \mathrm{P}$-value & 0.0008 & 0.0030 & 0.0024 & 0.0368 & 0.0303 \\
\hline \multicolumn{6}{|l|}{ Tolpyralate + atrazine treatments } \\
\hline No tank mix partner & 42 & 41 & 41 & 6.0 & 24.39 \\
\hline Tolpyralate + Atrazine & 89 & 91 & 91 & 0.8 & 1.03 \\
\hline Tolpyralate + Atrazine $+\mathrm{MSO}^{\mathrm{d}}$ & 98 & 99 & 98 & 0.5 & 0.09 \\
\hline Tolpyralate + Atrazine $+\mathrm{UAN}^{\mathrm{e}}$ & 91 & 92 & 93 & 0.4 & 0.18 \\
\hline Tolpyralate + Atrazine $+\mathrm{MSO}+\mathrm{UAN}$ & 98 & 99 & 98 & 0.3 & 0.14 \\
\hline $\mathrm{SE}^{\mathrm{b}}$ & 2 & 3 & 2 & 0.4 & 2.47 \\
\hline Tolpyralate + atrazine P-value & $<0.0001$ & $<0.0001$ & $<0.0001$ & 0.0001 & 0.0003 \\
\hline \multicolumn{6}{|l|}{ Interaction } \\
\hline $\begin{array}{c}\text { Roundup WeatherMAX }{ }^{\otimes \star} \text { tolpyralate }+ \\
\text { atrazine P-value }\end{array}$ & $<0.0001$ & $<0.0001$ & $<0.0001$ & 0.0017 & 0.0005 \\
\hline
\end{tabular}

${ }^{\mathrm{a}} \mathrm{WAA}$, weeks after treatment application. ${ }^{\mathrm{b}}$ Standard error of the mean. ${ }^{\mathrm{C}}$ Tolpyralate applied at $30 \mathrm{~g} \cdot \mathrm{ai} \cdot \mathrm{ha} \mathrm{a}^{-1}$; atrazine applied at $560 \mathrm{~g} \cdot \mathrm{ai} \cdot \mathrm{ha}^{-1} .{ }^{\mathrm{d}} \mathrm{MSO}, 0.50 \% \mathrm{v} / \mathrm{v} .{ }^{\mathrm{e}} \mathrm{UAN}, 2.50 \% \mathrm{v} / \mathrm{v}$. ${ }^{\mathrm{f}}$ Density and biomass collected 8 WAA. Means within column followed by the same lowercase letter are not statistically different according to Tukey-Kramer's LSD $(P=0.05)$.

in pigweed control from the addition of UAN to tolpyralate + atrazine + MSO. Tolpyralate + atrazine reduced pigweed density and biomass $88 \%$ and $97 \%$, respectively; there was no further decrease in pigweed density and biomass with the addition of an adjuvant to tolpyralate + atrazine. Metzger et al. 2018 [27] reported that pigweed could be controlled with lower than labelled doses of tolpyralate + atrazine, suggesting a high level of pigweed sensitivity to tolpyralate + atrazine; tolpyralate (30 and $\left.40 \mathrm{~g} \cdot \mathrm{ai} \cdot \mathrm{ha}^{-1}\right)+$ atrazine $\left(1000 \mathrm{~g} \cdot \mathrm{ai} \cdot \mathrm{ha}^{-1}\right)$ averaged over PRE and POST applications controlled Powell amaranth, a pigweed species $88 \%-93 \%$ [9]. Roundup WeatherMAX ${ }^{\circledast}$ controlled pigweed $\geq 96 \%$ at 2,4 and 8 WAA; there was no improvement in pigweed control with the addition of tolpyralate + atrazine, regardless of adjuvants. Roundup WeatherMAX ${ }^{\oplus}$ reduced pigweed density and biomass $\geq 96 \%$, there further decrease in pigweed density and biomass from the addition of tolpyralate + atrazine, with or without adjuvants, to Roundup WeatherMAX ${ }^{\oplus}$. No previous research has evaluated Roundup WeatherMAX ${ }^{\oplus}$ plus tolpyralate + atrazine tank mix effects on pigweed control; however, Starke and Oliver 1998 [9] reported that tank mixtures of Roundup 
Table 5. Pigweed (Amaranthus spp. [AMASS]) control 2, 4, and 8 WAA, density and biomass in corn with Roundup WeatherMAX ${ }^{\circledast}$ plus tolpyralate plus atrazine from five field experiments conducted in Ontario in 2018 and 2019.

\begin{tabular}{|c|c|c|c|}
\hline & \multicolumn{3}{|c|}{ Roundup WeatherMAX $\left(\mathrm{g} \cdot \mathrm{ae} \cdot \mathrm{ha}^{-1}\right)$} \\
\hline & 0 & 900 & SE \\
\hline \multicolumn{4}{|l|}{ Visible Weed Control 2 WAA $A^{a}(\%)$} \\
\hline No tank mix partner & $0 c \mathrm{c}$ & $97 a Z$ & 8 \\
\hline Tolpyralate + Atrazine $^{\mathrm{b}}$ & $77 \mathrm{bY}$ & $98 \mathrm{aZ}$ & 4 \\
\hline Tolpyralate + Atrazine $+\mathrm{MSO}^{c}$ & $98 \mathrm{aZ}$ & $98 \mathrm{aZ}$ & 0.7 \\
\hline Tolpyralate + Atrazine + UAN $^{d}$ & $80 \mathrm{bY}$ & $98 \mathrm{aZ}$ & 3 \\
\hline Tolpyralate + Atrazine $+\mathrm{MSO}+\mathrm{UAN}$ & $98 \mathrm{aZ}$ & $99 a Z$ & 0.5 \\
\hline $\mathrm{SE}^{\mathrm{e}}$ & 4 & 0.3 & \\
\hline \multicolumn{4}{|l|}{ Visible Weed Control 4 WAA (\%) } \\
\hline No tank mix partner & $0 \mathrm{cY}$ & $96 a Z$ & 8 \\
\hline Tolpyralate + Atrazine & $77 \mathrm{bY}$ & $98 \mathrm{aZ}$ & 5 \\
\hline Tolpyralate + Atrazine + MSO & $98 \mathrm{aZ}$ & $99 \mathrm{aZ}$ & 0.5 \\
\hline Tolpyralate + Atrazine + UAN & $79 \mathrm{bY}$ & $99 \mathrm{aZ}$ & 4 \\
\hline Tolpyralate + Atrazine $+\mathrm{MSO}+\mathrm{UAN}$ & $99 \mathrm{aZ}$ & $99 \mathrm{aZ}$ & 0.5 \\
\hline SE & 4 & 0.4 & \\
\hline \multicolumn{4}{|l|}{ Visible Weed Control 8 WAA (\%) } \\
\hline No tank mix partner & $0 \mathrm{cY}$ & $96 a Z$ & 7 \\
\hline Tolpyralate + Atrazine & $77 \mathrm{bY}$ & $99 \mathrm{aZ}$ & 4 \\
\hline Tolpyralate + Atrazine $+\mathrm{MSO}$ & $97 \mathrm{aZ}$ & $99 \mathrm{aZ}$ & 0.9 \\
\hline Tolpyralate + Atrazine + UAN & $80 \mathrm{bY}$ & $99 a Z$ & 4 \\
\hline Tolpyralate + Atrazine + MSO + UAN & $98 \mathrm{aZ}$ & $99 \mathrm{aZ}$ & 0.6 \\
\hline SE & 4 & 0.4 & \\
\hline \multicolumn{4}{|l|}{$\operatorname{Density}\left(\right.$ no $\left.\mathrm{m}^{-2}\right)$} \\
\hline No tank mix partner & $15.79 \mathrm{aY}$ & $0.53 \mathrm{aZ}$ & 1.98 \\
\hline Tolpyralate + Atrazine & $1.84 \mathrm{bZ}$ & $0.14 \mathrm{aZ}$ & 0.36 \\
\hline Tolpyralate + Atrazine $+\mathrm{MSO}$ & $0.92 \mathrm{bZ}$ & $0.12 \mathrm{aZ}$ & 0.19 \\
\hline Tolpyralate + Atrazine + UAN & $0.89 \mathrm{bZ}$ & $0.10 \mathrm{aZ}$ & 0.24 \\
\hline Tolpyralate + Atrazine $+\mathrm{MSO}+\mathrm{UAN}$ & $0.28 \mathrm{bZ}$ & $0.26 \mathrm{aZ}$ & 0.13 \\
\hline SE & 0.85 & 0.071 & \\
\hline \multicolumn{4}{|l|}{ Biomass $\left(\mathrm{g} \cdot \mathrm{m}^{-2}\right)$} \\
\hline No tank mix partner & $90.02 \mathrm{aY}$ & $0.11 \mathrm{aZ}$ & 11.71 \\
\hline Tolpyralate + Atrazine & $2.69 \mathrm{bZ}$ & $0.01 \mathrm{aZ}$ & 0.97 \\
\hline Tolpyralate + Atrazine $+\mathrm{MSO}$ & $0.17 \mathrm{bZ}$ & $0.01 \mathrm{aZ}$ & 0.036 \\
\hline Tolpyralate + Atrazine + UAN & $0.36 \mathrm{bZ}$ & $0.01 \mathrm{aZ}$ & 0.13 \\
\hline Tolpyralate + Atrazine $+\mathrm{MSO}+\mathrm{UAN}$ & $0.095 \mathrm{bZ}$ & $0.18 \mathrm{aZ}$ & 0.089 \\
\hline SE & 4.871 & 0.035 & \\
\hline
\end{tabular}

${ }^{a}$ WAA, weeks after treatment application. ${ }^{\text {b} T o l p y r a l a t e ~ a p p l i e d ~ a t ~} 30 \mathrm{~g} \cdot \mathrm{ai} \cdot \mathrm{ha}^{-1}$; atrazine applied at 560 g.ai.ha ${ }^{-1}$. ${ }^{\mathrm{C} S S O}, 0.50 \% \mathrm{v} / \mathrm{v}$. ${ }^{\mathrm{d}} \mathrm{UAN}, 2.50 \% \mathrm{v} / \mathrm{v}$. ${ }^{\mathrm{e} S t a n d a r d}$ error of the mean. Means within column followed by the same lowercase letter, or means within row followed by the same uppercase letter, are not statistically different according to Tukey-Kramer's LSD $(P=0.05)$. 
WeatherMAX ${ }^{\circledast}$ plus herbicides such as fomesafen or sulfentrazone were antagonistic for control of Palmer amaranth, a pigweed species. Similar to results of this study, Starke and Oliver 1998 [9] found Roundup WeatherMAX ${ }^{\circledast}$ plus chlorimuron or imazethapyr improved Palmer amaranth control, having an additive effect. The addition of Roundup WeatherMAX ${ }^{\circledast}$ to tolpyralate + atrazine and tolpyralate + atrazine + UAN improved pigweed control by $19 \%-22 \%$; there was no increase in control from the addition of Roundup WeatherMAX ${ }^{\bowtie}$ to tolpyralate + atrazine when MSO was the adjuvant.

\subsection{Common Ragweed}

Variance analysis of common ragweed data indicated a Roundup WeatherMAX ${ }^{\circledast}$ by tolpyralate + atrazine plus adjuvant treatment interaction for weed control at 2, 4 and 8 WAA $(P<0.0001)$ and common ragweed biomass $(P=0.0007)$ (Table $6)$. There was not a significant interaction between Roundup WeatherMAX ${ }^{\circledast}$ and tolpyralate + atrazine for common ragweed density $(P=0.1035)$; however, for the tolpyralate + atrazine plus adjuvant treatment there was a significant main effect $(P<0.0001)$. Roundup WeatherMAX ${ }^{\oplus}$, averaged across all tolpyralate plus adjuvant treatments, did not decrease common ragweed density (Table 6). However, averaged across glyphosate at 0 and $900 \mathrm{~g} \cdot \mathrm{ae} \cdot \mathrm{ha}^{-1}$, tolpyralate + atrazine reduced common ragweed density $81 \%$ compared to the control; there was no further decrease in density with the addition of MSO, UAN or MSO + UAN to tolpyralate + atrazine.

Tolpyralate + atrazine controlled common ragweed $\geq 77 \%$ at 2,4 , and 8 WAA; there was no increase in common ragweed control with the addition of UAN (Table 7). The addition of MSO or MSO + UAN to tolpyralate + atrazine improved common ragweed control to $\geq 94 \%$. Tolpyralate + atrazine reduced common ragweed density $91 \%$, there was no further decrease in common ragweed density when MSO, UAN or MSO + UAN were added to tolpyralate + atrazine. Results of this study are similar to Metzger et al. 2019 [11] who reported $99 \%$ control of common ragweed 2 WAA when tolpyralate + atrazine (30 $+1000 \mathrm{~g} \cdot \mathrm{ai} \cdot \mathrm{ha}^{-1}$ ) plus recommended adjuvants was applied early POST. Similar to this study, Bunting et al. 2004 [28] found that the addition of MSO to foramsulfuron improved control of common ragweed. However, the addition of MSO, $\mathrm{UAN}$ or MSO + UAN to tolpyralate + atrazine did not increase common ragweed control 8 WAA or reduce common ragweed biomass. Roundup WeatherMAX ${ }^{\oplus}$ controlled common ragweed $88 \%-91 \%$ at 2, 4 and 8 WAA; control was not improved when tolpyralate + atrazine was added alone or in combination with MSO, UAN or MSO + UAN. Roundup WeatherMAX ${ }^{\circledR}$ reduced common ragweed biomass 99\%; biomass was not reduced further with the addition of tolpyralate + atrazine to Roundup WeatherMAX ${ }^{\circledast}$. The addition of Roundup WeatherMAX ${ }^{\circledast}$ to tolpyralate + atrazine improved control of common ragweed when applied alone and with UAN at 2 WAA and with tolpyralate + atrazine at 4 WAA. By 8 WAA, there was no increase in common ragweed control when Roundup WeatherMAX ${ }^{\circledast}$ was added to tolpyralate + atrazine. 
Table 6. Significance of main effects and interactions for common ragweed (Ambrosia artemisiifolia L.) control, density and biomass in corn with Roundup WeatherMAX ${ }^{\circledast}$ plus tolpyralate plus atrazine from six field experiments conducted in Ontario in 2018 and 2019.

\begin{tabular}{|c|c|c|c|c|c|}
\hline \multirow{2}{*}{ Main effects } & \multicolumn{3}{|c|}{ Visible Weed Control (\%) } & \multirow{2}{*}{$\begin{array}{l}\text { Density }^{\mathrm{f}} \\
\left(\text { no } \cdot \mathrm{m}^{-2}\right)\end{array}$} & \multirow{2}{*}{$\begin{array}{c}\text { Biomass }^{\mathrm{f}} \\
\left(\mathrm{g} \cdot \mathrm{m}^{-2}\right)\end{array}$} \\
\hline & $2 \mathrm{WAA}^{\mathrm{a}}$ & $4 \mathrm{WAA}$ & $8 \mathrm{WAA}$ & & \\
\hline \multicolumn{6}{|l|}{ Roundup WeatherMAX $X^{\oplus}\left(g \cdot a e h a^{-1}\right)$} \\
\hline 0 & 69 & 71 & 70 & $12 \mathrm{a}$ & 24 \\
\hline 900 & 93 & 94 & 94 & $5 a$ & 2 \\
\hline $\mathrm{SE}^{\mathrm{b}}$ & 2 & 2 & 2 & 2.17 & 4 \\
\hline Roundup WeatherMAX ${ }^{\circledast}$ P-value & $<0.0001$ & 0.0014 & 0.0025 & 0.4868 & 0.0991 \\
\hline \multicolumn{6}{|l|}{ Tolpyralate + atrazine treatments ${ }^{c}$} \\
\hline No tank mix partner & 33 & 34 & 35 & $42 a$ & 67.2 \\
\hline Tolpyralate + Atrazine & 85 & 86 & 87 & $8 b$ & 6.9 \\
\hline Tolpyralate + Atrazine $+\mathrm{MSO}^{\mathrm{d}}$ & 95 & 97 & 95 & $2 b$ & 0.6 \\
\hline Tolpyralate + Atrazine $+\mathrm{UAN}^{\mathrm{e}}$ & 88 & 90 & 90 & $5 b$ & 6.6 \\
\hline Tolpyralate + Atrazine + MSO + UAN & 96 & 97 & 95 & $2 b$ & 0.6 \\
\hline $\mathrm{SE}^{\mathrm{b}}$ & 2 & 2 & 2 & 2.17 & 4.03 \\
\hline Tolpyralate + atrazine P-value & $<0.0001$ & $<0.0001$ & $<0.0001$ & $<0.0001$ & 0.0011 \\
\hline \multicolumn{6}{|l|}{ Interaction } \\
\hline $\begin{array}{l}\text { Roundup WeatherMAX }{ }^{\otimes \star} \text { tolpyralate }+ \\
\text { atrazine } \mathrm{P} \text {-value }\end{array}$ & $<0.0001$ & $<0.0001$ & $<0.0001$ & 0.1035 & 0.0007 \\
\hline
\end{tabular}

${ }^{a}$ WAA, weeks after treatment application. ${ }^{\mathrm{b}}$ Standard error of the mean. ${ }^{\mathrm{c}}$ Tolpyralate applied at $30 \mathrm{~g} \cdot \mathrm{ai} \cdot \mathrm{ha} \mathrm{a}^{-1}$; atrazine applied at $560 \mathrm{~g} \cdot \mathrm{ai} \cdot \mathrm{ha}^{-1} .{ }^{\mathrm{d}} \mathrm{MSO}, 0.50 \% \mathrm{v} / \mathrm{v}$. ${ }^{\mathrm{e}} \mathrm{UAN}, 2.50 \% \mathrm{v} / \mathrm{v}$. ${ }^{\mathrm{f}}$ Density and biomass collected 8 WAA. Means within column followed by the same lowercase letter are not statistically different according to Tukey-Kramer's LSD $(P=0.05)$.

\subsection{Common Lambsquarters}

Data analysis indicated that there was a significant Roundup WeatherMAX ${ }^{\oplus}$ by tolpyralate + atrazine plus adjuvant interaction for lambsquarters weed control at 2,4 and 8 WAA $(P<0.0001)$, lambsquarters density $(P=0.0189)$ and biomass $(P<0.0001)$ (Table 8).

Tolpyralate + atrazine controlled lambsquarters $67 \%-70 \%$ at 2,4 and 8 WAA, there was no increase in control when UAN was added (Table 9). Tolpyralate + atrazine plus MSO controlled lambsquarters $\geq 95 \%$, there was no further increase in control when UAN was added to tolpyralate + atrazine + MSO. Tolpyralate + atrazine reduced lambsquarters density and biomass $66 \%$ and $89 \%$, respectively; there was no further decrease in density and biomass when UAN was added to tolpyralate + atrazine. Consistent with control ratings, tolpyralate + atrazine $+\mathrm{MSO}$ and tolpyralate + atrazine $+\mathrm{MSO}+$ UAN decreased lambsquarters density and biomass $\geq 99 \%$. Similar to results from this study, Bunting et al. 2004 [28] reported enhanced control of lambsquarters when MSO was added to foramsulfuron. Roundup WeatherMAX ${ }^{\circledR}$ controlled lambsquarters 
Table 7. Common ragweed (Ambrosia artemisiifolia L.) control 2, 4, and 8 WAA and biomass in corn with Roundup WeatherMAX ${ }^{\circledR}$ plus tolpyralate plus atrazine from six field experiments conducted in Ontario in 2018 and 2019.

\begin{tabular}{|c|c|c|c|}
\hline & \multicolumn{3}{|c|}{ Roundup WeatherMAX ${ }^{\infty}$ (g.ae $\left.\cdot \mathrm{ha}^{-1}\right)$} \\
\hline & 0 & 900 & SE \\
\hline \multicolumn{4}{|l|}{ Visible Weed Control $2 W A A^{a}(\%)$} \\
\hline No tank mix partner & $0 \mathrm{cY}$ & $88 \mathrm{aZ}$ & 6 \\
\hline Tolpyralate + Atrazine $^{\mathrm{b}}$ & $77 b Y$ & $92 \mathrm{aZ}$ & 2 \\
\hline Tolpyralate + Atrazine $+\mathrm{MSO}^{c}$ & $94 \mathrm{aZ}$ & $96 \mathrm{aZ}$ & 1 \\
\hline Tolpyralate + Atrazine $+\mathrm{UAN}^{\mathrm{d}}$ & $83 \mathrm{bY}$ & $93 \mathrm{aZ}$ & 2 \\
\hline Tolpyralate + Atrazine + MSO + UAN & $95 \mathrm{aZ}$ & $96 \mathrm{aZ}$ & 1 \\
\hline $\mathrm{SE}^{\mathrm{e}}$ & 3 & 0.6 & \\
\hline \multicolumn{4}{|l|}{ Visible Weed Control 4 WAA (\%) } \\
\hline No tank mix partner & $0 \mathrm{cY}$ & $89 \mathrm{aZ}$ & 6 \\
\hline Tolpyralate + Atrazine & $79 \mathrm{bY}$ & $92 \mathrm{aZ}$ & 3 \\
\hline Tolpyralate + Atrazine + MSO & $96 \mathrm{aZ}$ & $97 a Z$ & 1 \\
\hline Tolpyralate + Atrazine + UAN & $85 \mathrm{abZ}$ & $94 \mathrm{aZ}$ & 3 \\
\hline Tolpyralate + Atrazine $+\mathrm{MSO}+\mathrm{UAN}$ & $96 \mathrm{aZ}$ & $97 a Z$ & 1 \\
\hline SE & 4 & 0.7 & \\
\hline \multicolumn{4}{|l|}{ Visible Weed Control 8 WAA (\%) } \\
\hline No tank mix partner & obY & $91 \mathrm{aZ}$ & 6 \\
\hline Tolpyralate + Atrazine & $80 \mathrm{aZ}$ & $92 \mathrm{aZ}$ & 4 \\
\hline Tolpyralate + Atrazine + MSO & $94 \mathrm{aZ}$ & $96 \mathrm{aZ}$ & 1 \\
\hline Tolpyralate + Atrazine + UAN & $85 \mathrm{aZ}$ & $95 \mathrm{aZ}$ & 4 \\
\hline Tolpyralate + Atrazine $+\mathrm{MSO}+\mathrm{UAN}$ & $94 \mathrm{aZ}$ & $95 \mathrm{aZ}$ & 1 \\
\hline SE & 4 & 0.8 & \\
\hline \multicolumn{4}{|l|}{ Biomass $\left(\mathrm{g} \cdot \mathrm{m}^{-2}\right)$} \\
\hline No tank mix partner & $283.0 \mathrm{aY}$ & $2.0 \mathrm{aZ}$ & 15.2 \\
\hline Tolpyralate + Atrazine & $24.1 \mathrm{bZ}$ & $1.4 \mathrm{aZ}$ & 8.9 \\
\hline Tolpyralate + Atrazine + MSO & $0.7 \mathrm{bZ}$ & $0.6 \mathrm{aZ}$ & 0.2 \\
\hline Tolpyralate + Atrazine + UAN & $22.4 \mathrm{bZ}$ & $1.4 \mathrm{aZ}$ & 7.7 \\
\hline Tolpyralate + Atrazine + MSO + UAN & $0.5 \mathrm{bZ}$ & $0.7 \mathrm{aZ}$ & 0.2 \\
\hline SE & 7.8 & 0.2 & \\
\hline
\end{tabular}

${ }^{a}$ WAA, weeks after treatment application. ${ }^{\text {b}}$ Tolpyralate applied at $30 \mathrm{~g} \cdot \mathrm{ai}^{\mathrm{h}} \mathrm{ha}^{-1}$; atrazine applied at 560 g.ai $\cdot \mathrm{ha}^{-1} \cdot{ }^{\mathrm{c}} \mathrm{MSO}, 0.50 \% \mathrm{v} / \mathrm{v} .{ }^{\mathrm{d}} \mathrm{UAN}, 2.50 \% \mathrm{v} / \mathrm{v}$. ${ }^{\mathrm{e}}$ Standard error of the mean. Means within column followed by the same lowercase letter, or means within row followed by the same uppercase letter, are not statistically different according to Tukey-Kramer's LSD $(P=0.05)$.

$88 \%-91 \%$ at 2, 4 and 8 WAA. At 2 WAA the addition of tolpyralate + atrazine + MSO or tolpyralate + atrazine + MSO + UAN to Roundup WeatherMAX ${ }^{\varpi}$ improved lambsquarters control to $\geq 98 \%$. At 4 and 8 WAA after application, there was no increase in lambsquarters control when tolpyralate + atrazine was added to Roundup WeatherMAX ${ }^{\oplus}$. Roundup WeatherMAX ${ }^{\circledR}$ reduced lambsquarters density $70 \%$, there was no further decrease in lambsquarters density when tolpyralate + atrazine or tolpyralate + atrazine + UAN was added to Roundup WeatherMAX ${ }^{\oplus}$. In contrast, the addition of tolpyralate + atrazine + either MSO 
Table 8. Significance of main effects and interactions for lambsquarters (Chenopodium album L.) control, density and biomass in corn with Roundup WeatherMAX ${ }^{\circledast}$ plus tolpyralate plus atrazine from six field experiments conducted in Ontario in 2018 and 2019.

\begin{tabular}{|c|c|c|c|c|c|}
\hline \multirow{2}{*}{ Main effects } & \multicolumn{3}{|c|}{ Visible Weed Control (\%) } & \multirow{2}{*}{$\begin{array}{l}\text { Density }{ }^{f} \\
\left(\text { no } \cdot m^{-2}\right)\end{array}$} & \multirow{2}{*}{$\begin{array}{c}\text { Biomass }{ }^{\mathrm{f}} \\
\left(\mathrm{g} \cdot \mathrm{m}^{-2}\right)\end{array}$} \\
\hline & $2 \mathrm{WAA}^{\mathrm{a}}$ & 4 WAA & $8 \mathrm{WAA}$ & & \\
\hline \multicolumn{6}{|l|}{ Roundup WeatherMAX $X^{\oplus}\left(g \cdot a e h a^{-1}\right)$} \\
\hline 0 & 68 & 67 & 65 & 10.7 & 12.77 \\
\hline 900 & 96 & 97 & 96 & 2.7 & 0.55 \\
\hline $\mathrm{SE}^{\mathrm{b}}$ & 2 & 2 & 2 & 0.8 & 1.58 \\
\hline Roundup WeatherMAX ${ }^{\circledast}$ P-value & $<0.0001$ & 0.0006 & 0.0010 & 0.0546 & 0.0097 \\
\hline \multicolumn{6}{|l|}{ Tolpyralate + atrazine treatments } \\
\hline No tank mix partner & 35 & 33 & 33 & 21.9 & 33.34 \\
\hline Tolpyralate + Atrazine & 86 & 86 & 85 & 6.1 & 2.95 \\
\hline Tolpyralate + Atrazine + MSO $^{\mathrm{d}}$ & 98 & 98 & 97 & 1.3 & 0.30 \\
\hline Tolpyralate + Atrazine $+\mathrm{UAN}^{e}$ & 86 & 86 & 85 & 6.6 & 4.39 \\
\hline Tolpyralate + Atrazine + MSO + UAN & 98 & 98 & 97 & 1.2 & 0.25 \\
\hline $\mathrm{SE}^{\mathrm{b}}$ & 2 & 2 & 2 & 0.8 & 1.58 \\
\hline Tolpyralate + atrazine $\mathrm{P}$-value & $<0.0001$ & $<0.0001$ & $<0.0001$ & $<0.0001$ & $<0.0001$ \\
\hline \multicolumn{6}{|l|}{ Interaction } \\
\hline $\begin{array}{l}\text { Roundup WeatherMAX }{ }^{\star *} \text { tolpyralate }+ \\
\text { atrazine P-value }\end{array}$ & $<0.0001$ & $<0.0001$ & $<0.0001$ & 0.0189 & $<0.0001$ \\
\hline
\end{tabular}

${ }^{\mathrm{a}} \mathrm{WAA}$, weeks after treatment application. ${ }^{\mathrm{b}}$ Standard error of the mean. ${ }^{\mathrm{c}}$ Tolpyralate applied at $30 \mathrm{~g} \cdot \mathrm{ai} \cdot \mathrm{ha}^{-1}$; atrazine applied at 560 g.ai.ha ${ }^{-1} .{ }^{\mathrm{d}} \mathrm{MSO}, 0.50 \% \mathrm{v} / \mathrm{v} .{ }^{\mathrm{e}} \mathrm{UAN}, 2.50 \% \mathrm{v} / \mathrm{v}$. ${ }^{\mathrm{f}}$ Density and biomass collected 8 WAA. Means within column followed by the same lowercase letter are not statistically different according to Tukey-Kramer's LSD $(P=0.05)$.

or MSO + UAN to Roundup WeatherMAX ${ }^{\bullet}$ reduced lambsquarters density $\geq$ 95\%. Roundup WeatherMAX ${ }^{\oplus}$ reduced lambsquarters biomass $97 \%$, there was no further decrease in lambsquarters biomass when tolpyralate + atrazine was added to Roundup WeatherMAX ${ }^{\oplus}$. The addition of Roundup WeatherMAX ${ }^{ø}$ to tolpyralate + atrazine and tolpyralate + atrazine + UAN improved lambsquarters control at all evaluation timings and caused a further reduction in density and biomass. When MSO was the adjuvant with tolpyralate + atrazine, control was not improved from the addition of Roundup WeatherMAX ${ }^{\oplus}$.

\subsection{Ladysthumb}

Data analysis indicated that there was no interaction between Roundup WeatherMAX ${ }^{\odot}$ and tolpyralate + atrazine plus adjuvant treatment for ladysthumb density $(P=0.2773)$ or biomass $(P=0.0902)$ (Table 10$)$. There was no significant main effect of Roundup WeatherMAX ${ }^{\circledR}(P=0.1791)$ or tolpyralate + atrazine plus adjuvant $(P=0.0766)$ treatment for ladysthumb density. Averaged across all tolpyralate + atrazine plus adjuvant treatments, Roundup Weather$\mathrm{MAX}^{\oplus}$ did not influence ladysthumb biomass $(P=0.1123)$ (Table 10). Averaged across both levels of Roundup WeatherMAX ${ }^{\oplus}$, ladysthumb biomass was reduced 
Table 9. Common lambsquarters (Chenopodium album L.) control 2, 4, and 8 WAA, density and biomass in corn with Roundup WeatherMAX ${ }^{\circledR}$ plus tolpyralate plus atrazine from six field experiments conducted in Ontario in 2018 and 2019.

\begin{tabular}{|c|c|c|c|}
\hline & \multicolumn{2}{|c|}{ Roundup WeatherMAX ${ }^{\oplus}\left(\mathrm{g} \cdot \mathrm{ae} \cdot \mathrm{ha}^{-1}\right)$} & \multirow[b]{2}{*}{ SE } \\
\hline & 0 & 900 & \\
\hline \multicolumn{4}{|l|}{ Visible Weed Control 2 WA $A^{a}(\%)$} \\
\hline No tank mix partner & $0 \mathrm{cY}$ & $91 \mathrm{bZ}$ & 7 \\
\hline Tolpyralate + Atrazine $^{\mathrm{b}}$ & $70 \mathrm{bY}$ & 96abZ & 3 \\
\hline Tolpyralate + Atrazine $+\mathrm{MSO}^{c}$ & $97 a Z$ & $99 \mathrm{aZ}$ & 0.4 \\
\hline Tolpyralate + Atrazine + UAN $^{\mathrm{d}}$ & $72 b \mathrm{~b}$ & $96 a b Z$ & 3 \\
\hline Tolpyralate + Atrazine + MSO + UAN & $98 \mathrm{aZ}$ & $98 \mathrm{aZ}$ & 0.3 \\
\hline $\mathrm{SE}^{\mathrm{e}}$ & 3 & 1 & \\
\hline \multicolumn{4}{|l|}{ Visible Weed Control 4 WAA (\%) } \\
\hline No tank mix partner & $0 \mathrm{cY}$ & $88 \mathrm{aZ}$ & 6 \\
\hline Tolpyralate + Atrazine & $68 \mathrm{bY}$ & $98 \mathrm{aZ}$ & 4 \\
\hline Tolpyralate + Atrazine + MSO & $97 \mathrm{aZ}$ & $99 \mathrm{aZ}$ & 0.5 \\
\hline Tolpyralate + Atrazine + UAN & $72 b \mathrm{~b}$ & $97 a Z$ & 3 \\
\hline Tolpyralate + Atrazine $+\mathrm{MSO}+\mathrm{UAN}$ & $98 \mathrm{aZ}$ & $99 \mathrm{aZ}$ & 0.4 \\
\hline SE & 4 & 1 & \\
\hline \multicolumn{4}{|l|}{ Visible Weed Control 8 WAA (\%) } \\
\hline No tank mix partner & $0 \mathrm{cY}$ & $88 \mathrm{aZ}$ & 6 \\
\hline Tolpyralate + Atrazine & $67 \mathrm{bY}$ & $97 a Z$ & 4 \\
\hline Tolpyralate + Atrazine + MSO & $95 \mathrm{aZ}$ & $98 \mathrm{aZ}$ & 1 \\
\hline Tolpyralate + Atrazine + UAN & $70 \mathrm{bY}$ & $96 \mathrm{aZ}$ & 4 \\
\hline Tolpyralate + Atrazine + MSO + UAN & $97 \mathrm{aZ}$ & $98 \mathrm{aZ}$ & 1 \\
\hline SE & 4 & 1 & \\
\hline \multicolumn{4}{|l|}{$\operatorname{Density}\left(\mathrm{no} \cdot \mathrm{m}^{-2}\right)$} \\
\hline No tank mix partner & $31.05 \mathrm{aY}$ & $9.37 \mathrm{aZ}$ & 2.91 \\
\hline Tolpyralate + Atrazine & $10.67 \mathrm{bY}$ & $2.35 \mathrm{abZ}$ & 1.10 \\
\hline Tolpyralate + Atrazine + MSO & $2.13 c Z$ & $0.70 \mathrm{bZ}$ & 0.46 \\
\hline Tolpyralate + Atrazine + UAN & $12.08 \mathrm{bY}$ & $2.02 \mathrm{abZ}$ & 1.12 \\
\hline Tolpyralate + Atrazine $+\mathrm{MSO}+\mathrm{UAN}$ & $0.93 \mathrm{cZ}$ & $1.55 \mathrm{bZ}$ & 0.61 \\
\hline SE & 1.33 & 0.72 & \\
\hline \multicolumn{4}{|l|}{$\operatorname{Biomass}\left(\mathrm{g} \cdot \mathrm{m}^{-2}\right)$} \\
\hline No tank mix partner & $62.26 \mathrm{aY}$ & $1.69 \mathrm{aZ}$ & 6.93 \\
\hline Tolpyralate + Atrazine & 7..07bY & $0.44 \mathrm{aZ}$ & 1.22 \\
\hline Tolpyralate + Atrazine $+\mathrm{MSO}$ & $0.52 c Z$ & $0.10 \mathrm{aZ}$ & 0.16 \\
\hline Tolpyralate + Atrazine + UAN & $12.20 \mathrm{bY}$ & $0.44 \mathrm{aZ}$ & 1.57 \\
\hline Tolpyralate + Atrazine $+\mathrm{MSO}+\mathrm{UAN}$ & $0.19 \mathrm{cZ}$ & $0.32 \mathrm{aZ}$ & 0.10 \\
\hline SE & 3.04 & 0.10 & \\
\hline
\end{tabular}

${ }^{\mathrm{a} W A A}$, weeks after treatment application. ${ }^{\mathrm{b}}$ Tolpyralate applied at $30 \mathrm{~g} \cdot \mathrm{ai} \cdot \mathrm{ha}^{-1}$; atrazine applied at 560 g.ai.ha ${ }^{-1}$. ${ }^{\mathrm{c}} \mathrm{MSO}, 0.50 \% \mathrm{v} / \mathrm{v}$. ${ }^{\mathrm{d}} \mathrm{UAN}, 2.50 \% \mathrm{v} / \mathrm{v}$. ${ }^{\mathrm{e}} \mathrm{Standard}$ error of the mean. Means within column followed by the same lowercase letter, or means within row followed by the same uppercase letter, are not statistically different according to Tukey-Kramer's LSD $(P=0.05)$. 
Table 10. Significance of main effects and interactions for ladysthumb (Persicaria maculosa Gray) control, density and biomass in corn with Roundup WeatherMAX ${ }^{\circledast}$ plus tolpyralate plus atrazine from five field experiments conducted in Ontario in 2018 and 2019.

\begin{tabular}{|c|c|c|c|c|c|}
\hline \multirow{2}{*}{ Main effects } & \multicolumn{3}{|c|}{ Visible Weed Control (\%) } & \multirow{2}{*}{$\begin{array}{l}\text { Density }^{\mathrm{f}} \\
\left(\text { no } \cdot \mathrm{m}^{-2}\right)\end{array}$} & \multirow{2}{*}{$\begin{array}{c}\text { Biomass } \\
\left(\mathrm{g} \cdot \mathrm{m}^{-2}\right)\end{array}$} \\
\hline & $2 \mathrm{WAA}^{\mathrm{a}}$ & $4 \mathrm{WAA}$ & $8 \mathrm{WAA}$ & & \\
\hline \multicolumn{6}{|l|}{ Roundup WeatherMAX $\left(g \cdot a e h a^{-1}\right)$} \\
\hline 0 & 73 & 69 & 70 & $2 \mathrm{a}$ & 1a \\
\hline 900 & 97 & 98 & 98 & $0.5 \mathrm{a}$ & $0.1 \mathrm{a}$ \\
\hline $\mathrm{SE}^{\mathrm{b}}$ & 2 & 3 & 3 & 0.26 & 0.494 \\
\hline Roundup WeatherMAX ${ }^{\circledast} \mathrm{P}$-value & 0.0060 & 0.0126 & 0.0133 & 0.1791 & 0.1123 \\
\hline \multicolumn{6}{|l|}{ Tolpyralate + atrazine treatments ${ }^{c}$} \\
\hline No tank mix partner & 38 & 39 & 40 & $2 \mathrm{a}$ & $2 \mathrm{a}$ \\
\hline Tolpyralate + Atrazine & 91 & 91 & 91 & 1a & $0.6 \mathrm{ab}$ \\
\hline Tolpyralate + Atrazine + MSO $^{\mathrm{d}}$ & 97 & 96 & 97 & $0.8 \mathrm{a}$ & $0.3 \mathrm{~b}$ \\
\hline Tolpyralate + Atrazine $+\mathrm{UAN}^{\mathrm{e}}$ & 94 & 92 & 92 & $1 \mathrm{a}$ & $0.5 \mathrm{ab}$ \\
\hline Tolpyralate + Atrazine + MSO + UAN & 98 & 97 & 98 & $0.6 \mathrm{a}$ & $0.2 \mathrm{~b}$ \\
\hline $\mathrm{SE}^{\mathrm{b}}$ & 2 & 3 & 3 & 0.26 & \\
\hline Tolpyralate + atrazine $\mathrm{P}$-value & $<0.0001$ & $<0.0001$ & $<0.0001$ & 0.0766 & 0.0194 \\
\hline \multicolumn{6}{|l|}{ Interaction } \\
\hline $\begin{array}{c}\text { Roundup WeatherMAX }{ }^{\otimes *} \text { tolpyralate }+ \\
\text { atrazine } \mathrm{P} \text {-value }\end{array}$ & $<0.0001$ & $<0.0001$ & $<0.0001$ & 0.2773 & 0.0902 \\
\hline
\end{tabular}

${ }^{a}$ WAA, weeks after treatment application. ${ }^{\mathrm{b}}$ Standard error of the mean. ${ }^{\mathrm{c}}$ Tolpyralate applied at $30 \mathrm{~g} \cdot \mathrm{ai} \cdot \mathrm{ha} \mathrm{a}^{-1}$; atrazine applied at $560 \mathrm{~g} \cdot \mathrm{ai} \cdot \mathrm{ha}^{-1} .{ }^{\mathrm{d}} \mathrm{MSO}, 0.50 \% \mathrm{v} / \mathrm{v} .{ }^{\mathrm{e}} \mathrm{UAN}, 2.50 \% \mathrm{v} / \mathrm{v}$. ${ }^{\mathrm{f}}$ Density and biomass collected 8 WAA. Means within column followed by the same lowercase letter are not statistically different according to Tukey-Kramer's LSD $(P=0.05)$.

with tolpyralate + atrazine + MSO or MSO + UAN $(P=0.0194)$. Ladysthumb control varied with Roundup WeatherMAX ${ }^{\oplus}$ and tolpyralate + atrazine plus adjuvant treatment, producing a significant Roundup WeatherMAX ${ }^{\odot}$ by tolpyralate + atrazine interaction for weed control at 2,4 and 8 WAA $(P<0.001)$ (Table 10).

At 2, 4 and 8 WAA, tolpyralate + atrazine controlled ladysthumb $\geq 79 \%$ (Table 11). There was no further increase in control with the addition of MSO, $\mathrm{UAN}$ or MSO + UAN to tolpyralate + atrazine at 2 and $4 \mathrm{WAA}$; in contrast, at 8 WAA ladysthumb control with tolpyralate + atrazine was improved $17 \%$ with the addition of MSO + UAN. Similar to results of this study, Metzger et al. 2018 [27] reported that tolpyralate + atrazine + MSO + UAN controlled ladysthumb $\geq$ $90 \%$ at 2, 4 and 8 WAA. Roundup WeatherMAX ${ }^{\circledast}$ controlled ladysthumb $\geq 94 \%$ at all evaluation timings, there was no improvement in ladysthumb control when tolpyralate + atrazine was added to Roundup WeatherMAX ${ }^{\oplus}$. The addition of Roundup WeatherMAX ${ }^{\circledast}$ to tolpyralate + atrazine and tolpyralate + atrazine + UAN improved ladysthumb control at 2, 4 and 8 WAA; there was no increase in 
Table 11. Ladysthumb (Persicaria maculosa Gray) control 2, 4, and 8 WAA in corn with Roundup WeatherMAX ${ }^{\circledast}$ plus tolpyralate plus atrazine from five experiments conducted in Ontario in 2018 and 2019.

\begin{tabular}{|c|c|c|c|}
\hline & \multicolumn{3}{|c|}{ Roundup WeatherMAX $\left(\mathrm{g} \cdot \mathrm{ae} \cdot \mathrm{ha}^{-1}\right)$} \\
\hline & 0 & 900 & SE \\
\hline \multicolumn{4}{|l|}{ Visible Weed Control2 WAA ${ }^{a}(\%)$} \\
\hline No tank mix partner & obY & $94 \mathrm{aZ}$ & 8 \\
\hline Tolpyralate + Atrazine $^{\mathrm{b}}$ & $83 \mathrm{aY}$ & $97 \mathrm{aZ}$ & 3 \\
\hline Tolpyralate + Atrazine $+\mathrm{MSO}^{\mathrm{c}}$ & $96 a Z$ & $98 \mathrm{aZ}$ & 0.8 \\
\hline Tolpyralate + Atrazine + UAN $^{\mathrm{d}}$ & $88 \mathrm{aY}$ & $98 \mathrm{aZ}$ & 3 \\
\hline Tolpyralate + Atrazine + MSO + UAN & $97 a Z$ & $99 \mathrm{aZ}$ & 0.6 \\
\hline $\mathrm{SE}^{e}$ & 4 & 0.5 & \\
\hline \multicolumn{4}{|l|}{ Visible Weed Control 4 WAA (\%) } \\
\hline No tank mix partner & obY & $95 \mathrm{aZ}$ & 8 \\
\hline Tolpyralate + Atrazine & $79 \mathrm{aY}$ & $98 \mathrm{aZ}$ & 4 \\
\hline Tolpyralate + Atrazine + MSO & $92 \mathrm{aZ}$ & $98 \mathrm{aZ}$ & 1 \\
\hline Tolpyralate + Atrazine + UAN & $84 \mathrm{aY}$ & $98 \mathrm{aZ}$ & 4 \\
\hline Tolpyralate + Atrazine + MSO + UAN & $95 \mathrm{aZ}$ & $98 \mathrm{aZ}$ & 1 \\
\hline SE & 4 & 0.4 & \\
\hline \multicolumn{4}{|l|}{ Visible Weed Control 8 WAA (\%) } \\
\hline No tank mix partner & $0 \mathrm{cY}$ & $95 \mathrm{aZ}$ & 8 \\
\hline Tolpyralate + Atrazine & $79 b \mathrm{~b}$ & $98 \mathrm{aZ}$ & 4 \\
\hline Tolpyralate + Atrazine + MSO & $94 \mathrm{abZ}$ & $99 \mathrm{aZ}$ & 1 \\
\hline Tolpyralate + Atrazine + UAN & $83 \mathrm{abY}$ & $98 \mathrm{aZ}$ & 4 \\
\hline Tolpyralate + Atrazine + MSO + UAN & $96 a Z$ & $98 \mathrm{aZ}$ & 0.9 \\
\hline SE & 4 & 0.5 & \\
\hline
\end{tabular}

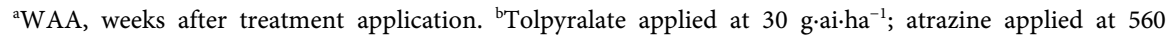
g.ai.ha ${ }^{-1}$. ${ }^{\mathrm{c}} \mathrm{MSO}, 0.50 \% \mathrm{v} / \mathrm{v}$. ${ }^{\mathrm{d}} \mathrm{UAN}, 2.50 \% \mathrm{v} / \mathrm{v}$. ${ }^{\mathrm{e}} \mathrm{Standard}$ error of the mean. Means within column followed by the same lowercase letter, or means within row followed by the same uppercase letter, are not statistically different according to Tukey-Kramer's LSD $(P=0.05)$.

ladysthumb control when Roundup WeatherMAX ${ }^{\circledR}$ was added to tolpyralate + atrazine $+\mathrm{MSO}$ or MSO + UAN.

\subsection{Wild Mustard}

Data analysis concluded that the Roundup WeatherMAX ${ }^{\circledR}$ by tolpyralate + atrazine interaction was not significant for wild mustard density $(P=0.5125)$ or biomass $(P=0.1336)$. Averaged across all levels of each factor, Roundup WeatherMAX ${ }^{\curvearrowleft}$ or tolpyralate + atrazine plus adjuvant treatment was there not a significant main effect for density or biomass (Table 12). There was a significant Roundup WeatherMAX ${ }^{\oplus}$ by tolpyralate + atrazine interaction for wild mustard control at $2(P=0.0011), 4(P=0.0080)$ and $8(P=0.0006)$ WAA (Table 12$)$.

Tolpyralate + atrazine controlled wild mustard 74,76 and $97 \%$ at 2,4 and 8 WAA, respectively (Table 13). There was no improvement in wild mustard 
Table 12. Significance of main effects and interactions for wild mustard (Sinapis arvensis L.) control, density and biomass in corn with Roundup WeatherMAX ${ }^{\circledast}$ plus tolpyralate plus atrazine from two field experiments conducted in Ontario in 2018 and 2019.

\begin{tabular}{|c|c|c|c|c|c|}
\hline \multirow{2}{*}{ Main effects } & \multicolumn{3}{|c|}{ Visible Weed Control (\%) } & \multirow{2}{*}{$\begin{array}{l}\text { Density } \\
\left(\text { no } \cdot m^{-2}\right)\end{array}$} & \multirow{2}{*}{$\begin{array}{c}\text { Biomass }^{\mathrm{f}} \\
\left(\mathrm{g} \cdot \mathrm{m}^{-2}\right)\end{array}$} \\
\hline & $2 \mathrm{WAA}^{\mathrm{a}}$ & $4 \mathrm{WAA}$ & $8 \mathrm{WAA}$ & & \\
\hline \multicolumn{6}{|l|}{ Roundup WeatherMAX $\left(g \cdot a e h a^{-1}\right)$} \\
\hline 0 & 65 & 67 & 79 & $13.67 \mathrm{a}$ & $3.66 \mathrm{a}$ \\
\hline 900 & 94 & 98 & 95 & $22.38 \mathrm{a}$ & $1.55 \mathrm{a}$ \\
\hline $\mathrm{SE}^{\mathrm{b}}$ & 3 & 3 & 3 & 5 & 1 \\
\hline Roundup WeatherMAX ${ }^{\circledast} \mathrm{P}$-value & 0.0982 & 0.1936 & 0.1453 & 0.6841 & 0.3607 \\
\hline \multicolumn{6}{|l|}{ Tolpyralate + atrazine treatments ${ }^{\mathcal{C}}$} \\
\hline No tank mix partner & 48 & 40 & 36 & $44.95 \mathrm{a}$ & $11.44 \mathrm{a}$ \\
\hline Tolpyralate + Atrazine & 83 & 90 & 97 & $22.51 \mathrm{a}$ & $2.14 \mathrm{a}$ \\
\hline Tolpyralate + Atrazine $+\mathrm{MSO}^{\mathrm{d}}$ & 91 & 95 & 95 & $19.21 \mathrm{a}$ & $1.52 \mathrm{a}$ \\
\hline Tolpyralate + Atrazine + UAN $^{e}$ & 84 & 92 & 96 & $5.51 \mathrm{a}$ & $0.55 \mathrm{a}$ \\
\hline Tolpyralate + Atrazine $+\mathrm{MSO}+\mathrm{UAN}$ & 91 & 96 & 95 & $9.62 \mathrm{a}$ & $1.17 \mathrm{a}$ \\
\hline $\mathrm{SE}^{\mathrm{b}}$ & 3 & 3 & 3 & 5 & 1 \\
\hline Tolpyralate + atrazine $\mathrm{P}$-value & 0.0050 & 0.0152 & 0.0004 & 0.2980 & 0.0913 \\
\hline \multicolumn{6}{|l|}{ Interaction } \\
\hline $\begin{array}{c}\text { Roundup WeatherMAX }{ }^{\otimes \star} \text { tolpyralate }+ \\
\text { atrazine P-value }\end{array}$ & 0.0011 & 0.0080 & 0.0006 & 0.5125 & 0.1336 \\
\hline
\end{tabular}

${ }^{a}$ WAA, weeks after treatment application. ${ }^{\text {b}}$ Standard error of the mean. ${ }^{\mathrm{C}}$ Tolpyralate applied at $30 \mathrm{~g} \cdot \mathrm{ai} \cdot \mathrm{ha} \mathrm{a}^{-1}$; atrazine applied at $560 \mathrm{~g} \cdot \mathrm{ai} \cdot \mathrm{ha}^{-1} .{ }^{\mathrm{d}} \mathrm{MSO}, 0.50 \% \mathrm{v} / \mathrm{v}$. ${ }^{\mathrm{e}} \mathrm{UAN}, 2.50 \% \mathrm{v} / \mathrm{v}$. ${ }^{\mathrm{f}}$ Density and biomass collected 8 WAA. Means within column followed by the same lowercase letter are not statistically different according to Tukey-Kramer's LSD $(P=0.05)$.

control with tolpyralate + atrazine with the addition of MSO, UAN or MSO + UAN. Metzger et al. 2018 [8] reported that tolpyralate alone provided poor control of wild mustard, however the addition of atrazine improved control to $\geq$ $90 \%$. Roundup WeatherMAX ${ }^{\oplus}$ controlled wild mustard $\geq 92 \%$ at 2,4 and 8 WAA; there was no improvement in wild mustard control when tolpyralate + atrazine was added to Roundup WeatherMAX ${ }^{\oplus}$. The addition of Roundup WeatherMAX ${ }^{ø}$ to tolpyralate + atrazine and tolpyralate + atrazine + UAN improved control of wild mustard 2 WAA. At 4 and 8 WAA, the addition of Roundup WeatherMAX ${ }^{\circledast}$ to tolpyralate + atrazine did not increase wild mustard control.

\subsection{Flower-of-an-Hour}

Data analysis indicated the Roundup WeatherMAX ${ }^{\oplus}$ by tolpyralate + atrazine interaction was not significant for flower-of-an-hour density $(P=0.4828)$ or biomass (0.4898) (Table 14). Averaged across all levels of tolpyralate + atrazine plus adjuvant, the addition of Roundup WeatherMAX ${ }^{\circledR}$ did not influence density or biomass. Similarly, averaged across 0 and $900 \mathrm{~g}$ ae ha $\mathrm{ha}^{-1}$ of Roundup WeatherMAX ${ }^{\oplus}$, the various tolpyralate + atrazine plus adjuvant treatments did not 
Table 13. Wild mustard (Sinapis arvensis L.) control 2, 4, and 8 WAA in corn with Roundup WeatherMAX ${ }^{\circledast}$ plus tolpyralate plus atrazine from two field experiments conducted in Ontario in 2018 and 2019.

\begin{tabular}{|c|c|c|c|}
\hline & \multicolumn{3}{|c|}{ Roundup WeatherMAX $\left(\mathrm{g} \cdot \mathrm{ae} \cdot \mathrm{ha}^{-1}\right)$} \\
\hline & 0 & 900 & SE \\
\hline \multicolumn{4}{|l|}{ Visible Weed Control $2 W A A^{a}(\%)$} \\
\hline No tank mix partner & $0 \mathrm{bY}$ & $97 \mathrm{aZ}$ & 12 \\
\hline Tolpyralate + Atrazine $e^{b}$ & $74 \mathrm{aY}$ & $93 \mathrm{aZ}$ & 4 \\
\hline Tolpyralate + Atrazine $+\mathrm{MSO}^{\mathrm{c}}$ & $87 a Z$ & $96 a Z$ & 2 \\
\hline Tolpyralate + Atrazine + UAN $^{d}$ & $74 \mathrm{aY}$ & $93 \mathrm{aZ}$ & 4 \\
\hline Tolpyralate + Atrazine $+\mathrm{MSO}+\mathrm{UAN}$ & $89 a Z$ & $94 \mathrm{aZ}$ & 1 \\
\hline $\mathrm{SE}^{e}$ & 6 & 1 & \\
\hline \multicolumn{4}{|l|}{ Visible Weed Control 4 WAA (\%) } \\
\hline No tank mix partner & $0 \mathrm{bY}$ & $95 \mathrm{aZ}$ & 12 \\
\hline Tolpyralate + Atrazine & $76 a Z$ & $99 \mathrm{aZ}$ & 5 \\
\hline Tolpyralate + Atrazine + MSO & $90 \mathrm{aZ}$ & $99 \mathrm{aZ}$ & 2 \\
\hline Tolpyralate + Atrazine + UAN & $83 a Z$ & $98 \mathrm{aZ}$ & 4 \\
\hline Tolpyralate + Atrazine $+\mathrm{MSO}+\mathrm{UAN}$ & $95 \mathrm{aZ}$ & $98 \mathrm{aZ}$ & 1 \\
\hline SE & 6 & 1 & \\
\hline \multicolumn{4}{|l|}{ Visible Weed Control 8 WAA (\%) } \\
\hline No tank mix partner & obY & $92 \mathrm{aZ}$ & 12 \\
\hline Tolpyralate + Atrazine & $97 \mathrm{aZ}$ & $96 \mathrm{aZ}$ & 1 \\
\hline Tolpyralate + Atrazine $+\mathrm{MSO}$ & $94 \mathrm{aZ}$ & $96 a Z$ & 1 \\
\hline Tolpyralate + Atrazine + UAN & $97 a Z$ & $96 \mathrm{aZ}$ & 1 \\
\hline Tolpyralate + Atrazine $+\mathrm{MSO}+\mathrm{UAN}$ & $95 \mathrm{aZ}$ & $96 a Z$ & 2 \\
\hline SE & 6 & 1 & \\
\hline
\end{tabular}

${ }^{\mathrm{a}} \mathrm{WAA}$, weeks after treatment application. ${ }^{\mathrm{b}}$ Tolpyralate applied at $30 \mathrm{~g} \cdot \mathrm{ai} \cdot \mathrm{ha}^{-1}$; atrazine applied at 560 g.ai.ha ${ }^{-1}$. ${ }^{\mathrm{C} S S O}, 0.50 \% \mathrm{v} / \mathrm{v}$. ${ }^{\mathrm{d}} \mathrm{UAN}, 2.50 \% \mathrm{v} / \mathrm{v}$. ${ }^{\mathrm{e} S t a n d a r d}$ error of the mean. Means within column followed by the same lowercase letter, or means within row followed by the same uppercase letter, are not statistically different according to Tukey-Kramer's LSD $(P=0.05)$.

influence density of biomass. There was a significant Roundup WeatherMAX by tolpyralate + atrazine interaction for flower-of-an-hour control at $2(P=$ 0.0014), 4 (0.0086) and 8 (0.0105) WAA (Table 14).

Tolpyralate + atrazine controlled flower-of-an-hour $63 \%-75 \%$ at 2,4 and 8 WAA (Table 15). The addition of MSO + UAN to tolpyralate + atrazine increased flower-of-an-hour control $25 \%$ at 2 WAA, however, at 4 and 8 WAA there was no improvement in flower-of-an-hour control when MSO, UAN or MSO + UAN was added to tolpyralate + atrazine. Roundup WeatherMAX ${ }^{ø}$ controlled flower-of-an-hour $84 \%-86 \%$ at 2,4 and 8 WAA. There was no improvement in flower-of-an-hour control when tolpyralate + atrazine was added to Roundup WeatherMAX ${ }^{\oplus}$. At 2 WAA, the addition of Roundup WeatherMAX ${ }^{\star}$ 
Table 14. Significance of main effects and interactions for flower-of-an-hour (Hibiscus trionum L.) control, density and biomass in corn with Roundup WeatherMAX ${ }^{\circledast}$ plus tolpyralate plus atrazine from two field experiments conducted in Ontario in 2018 and 2019.

\begin{tabular}{|c|c|c|c|c|c|}
\hline \multirow{2}{*}{ Main effects } & \multicolumn{3}{|c|}{ Visible Weed Control (\%) } & \multirow{2}{*}{$\begin{array}{l}\text { Density }{ }^{\mathrm{f}} \\
\left(\mathrm{no} \cdot \mathrm{m}^{-2}\right)\end{array}$} & \multirow{2}{*}{$\begin{array}{c}\text { Biomass }{ }^{\mathrm{f}} \\
\left(\mathrm{g} \cdot \mathrm{m}^{-2}\right)\end{array}$} \\
\hline & $2 \mathrm{WAA}^{\mathrm{a}}$ & $4 \mathrm{WAA}$ & 8 WAA & & \\
\hline \multicolumn{6}{|l|}{ Roundup WeatherMAX $X^{\oplus}\left(g \cdot a e \cdot h a^{-1}\right)$} \\
\hline 0 & 57 & 63 & 58 & $15.4 \mathrm{a}$ & $4.6 \mathrm{a}$ \\
\hline 900 & 91 & 92 & 90 & $11.6 \mathrm{a}$ & $0.6 \mathrm{a}$ \\
\hline $\mathrm{SE}^{\mathrm{b}}$ & 3 & 3 & 3 & 1.9 & 0.5 \\
\hline Roundup WeatherMAX ${ }^{\circledast}$ P-value & 0.0453 & 0.2111 & 0.2580 & 0.8664 & 0.3223 \\
\hline \multicolumn{6}{|l|}{ Tolpyralate + atrazine treatments ${ }^{\mathcal{c}}$} \\
\hline No tank mix partner & 31 & 43 & 31 & $17.0 \mathrm{a}$ & $3.3 \mathrm{a}$ \\
\hline Tolpyralate + Atrazine & 78 & 82 & 84 & $15.1 \mathrm{a}$ & $2.5 \mathrm{a}$ \\
\hline Tolpyralate + Atrazine $+\mathrm{MSO}^{\mathrm{d}}$ & 90 & 89 & 85 & $16.9 \mathrm{a}$ & $2.9 \mathrm{a}$ \\
\hline Tolpyralate + Atrazine $+\mathrm{UAN}^{\mathrm{e}}$ & 83 & 83 & 84 & $11.6 \mathrm{a}$ & $7.9 \mathrm{a}$ \\
\hline Tolpyralate + Atrazine + MSO + UAN & 90 & 91 & 87 & $8.3 \mathrm{a}$ & $1.3 \mathrm{a}$ \\
\hline $\mathrm{SE}^{\mathrm{b}}$ & 3 & 3 & 3 & 1.9 & 0.5 \\
\hline Tolpyralate + atrazine P-value & 0.0005 & 0.0176 & 0.0218 & 0.6321 & 0.6307 \\
\hline \multicolumn{6}{|l|}{ Interaction } \\
\hline $\begin{array}{c}\text { Roundup WeatherMAX }{ }^{\circledast \star} \text { tolpyralate }+ \\
\text { atrazine } P \text {-value }\end{array}$ & 0.0014 & 0.0086 & 0.0105 & 0.4829 & 0.4898 \\
\hline
\end{tabular}

${ }^{a}$ WAA, weeks after treatment application. ${ }^{\mathrm{b}}$ Standard error of the mean. ${ }^{\mathrm{c}}$ Tolpyralate applied at $30 \mathrm{~g} \cdot \mathrm{ai} \cdot \mathrm{ha} \mathrm{a}^{-1}$; atrazine applied at $560 \mathrm{~g} \cdot \mathrm{ai} \cdot \mathrm{ha}^{-1} .{ }^{\mathrm{d}} \mathrm{MSO}, 0.50 \% \mathrm{v} / \mathrm{v} .{ }^{\mathrm{e}} \mathrm{UAN}, 2.50 \% \mathrm{v} / \mathrm{v}$. ${ }^{\mathrm{f}}$ Density and biomass collected 8 WAA. Means within column followed by the same lowercase letter are not statistically different according to Tukey-Kramer's LSD $(P=0.05)$.

to tolpyralate + atrazine, tolpyralate + atrazine $+\mathrm{MSO}$, and tolpyralate + atrazine + UAN improved flower-of-an-hour control 27, $11 \%$ and $21 \%$, respectively; however, there was no improvement in flower-of-an-hour control when Roundup WeatherMAX ${ }^{\oplus}$ was added to tolpyralate + atrazine at 4 and 8 WAA.

\subsection{Barnyardgrass}

Analysis of variance in barnyardgrass data concluded that the Roundup WeatherMAX ${ }^{\oplus}$ by tolpyralate + atrazine interaction was not significant for barnyardgrass density $(P=0.3385)$ (Table 16$)$. Averaged across all levels of each factor, neither Roundup WeatherMAX ${ }^{\circledast}$ or tolpyralate + atrazine plus adjuvant was a significant main effect for density. However, there was a Roundup WeatherMAX ${ }^{\oplus}$ by tolpyralate + atrazine interaction for barnyardgrass control at 2,4 and 8 WAA $(P<0.0001)$ and biomass $(P=0.0002)$ (Table 16$)$.

Tolpyralate + atrazine controlled barnyardgrass 33,34 and $50 \%$ at 2,4 and 8 WAA, respectively (Table 17). The addition of UAN to tolpyralate + atrazine did not improve barnyardgrass control, however, the addition of MSO or MSO + 
Table 15. Flower-of-an-hour (Hibiscus trionum L.) control 2, 4, and 8 WAA in corn with Roundup WeatherMAX ${ }^{\circledast}$ plus tolpyralate plus atrazine from two field experiments conducted in Ontario in 2018 and 2019.

\begin{tabular}{|c|c|c|c|}
\hline & \multicolumn{3}{|c|}{ Roundup WeatherMAX ${ }^{\oplus}$ (g.ae $\left.\cdot \mathrm{ha}^{-1}\right)$} \\
\hline & 0 & 900 & SE \\
\hline \multicolumn{4}{|l|}{ Visible Weed Control $2 W A A^{a}(\%)$} \\
\hline No tank mix partner & $0 \mathrm{cY}$ & $84 \mathrm{aZ}$ & 11 \\
\hline Tolpyralate + Atrazine ${ }^{\mathrm{b}}$ & $63 b y$ & $90 \mathrm{aZ}$ & 4 \\
\hline Tolpyralate + Atrazine $+\mathrm{MSO}^{c}$ & $84 \mathrm{abY}$ & $95 a Z$ & 3 \\
\hline Tolpyralate + Atrazine + UAN $^{d}$ & $71 \mathrm{abY}$ & $92 \mathrm{aZ}$ & 4 \\
\hline Tolpyralate + Atrazine $+\mathrm{MSO}+\mathrm{UAN}$ & $88 \mathrm{aZ}$ & $92 \mathrm{aZ}$ & 3 \\
\hline $\mathrm{SE}^{\mathrm{e}}$ & 5 & 1 & \\
\hline \multicolumn{4}{|l|}{ Visible Weed Control 4 WAA (\%) } \\
\hline No tank mix partner & obY & $86 a Z$ & 11 \\
\hline Tolpyralate + Atrazine & $70 \mathrm{aZ}$ & $94 \mathrm{aZ}$ & 5 \\
\hline Tolpyralate + Atrazine + MSO & $82 \mathrm{aZ}$ & $95 a Z$ & 2 \\
\hline Tolpyralate + Atrazine + UAN & $74 \mathrm{aZ}$ & $92 \mathrm{aZ}$ & 5 \\
\hline Tolpyralate + Atrazine $+\mathrm{MSO}+\mathrm{UAN}$ & $89 \mathrm{aZ}$ & $93 a Z$ & 1 \\
\hline SE & 6 & 1 & \\
\hline \multicolumn{4}{|l|}{ Visible Weed Control 8 WAA (\%) } \\
\hline No tank mix partner & obY & $84 \mathrm{aZ}$ & 11 \\
\hline Tolpyralate + Atrazine & $75 \mathrm{aZ}$ & $92 \mathrm{aZ}$ & 6 \\
\hline Tolpyralate + Atrazine + MSO & $77 \mathrm{aZ}$ & $92 \mathrm{aZ}$ & 4 \\
\hline Tolpyralate + Atrazine + UAN & 79aZ & $89 \mathrm{aZ}$ & 4 \\
\hline Tolpyralate + Atrazine $+\mathrm{MSO}+\mathrm{UAN}$ & $82 \mathrm{aZ}$ & $92 \mathrm{aZ}$ & 3 \\
\hline SE & 6 & 1 & \\
\hline
\end{tabular}

${ }^{a}$ WAA, weeks after treatment application. ${ }^{\mathrm{b}}$ Tolpyralate applied at $30 \mathrm{~g} \cdot \mathrm{ai} \cdot \mathrm{ha}^{-1}$; atrazine applied at 560 g.ai $\mathrm{ha}^{-1}$. ${ }^{\mathrm{c}} \mathrm{MSO}, 0.50 \% \mathrm{v} / \mathrm{v}$. ${ }^{\mathrm{d}} \mathrm{UAN}, 2.50 \% \mathrm{v} / \mathrm{v}$. ${ }^{\mathrm{e} S t a n d a r d}$ error of the mean. Means within column followed by the same lowercase letter, or means within row followed by the same uppercase letter, are not statistically different according to Tukey-Kramer's LSD $(P=0.05)$.

UAN to tolpyralate + atrazine improved barnyardgrass control to $\geq 91, \geq 89$ and $84 \%$ at 2, 4 and 8 WAA, respectively. Tolpyralate + atrazine and tolpyralate + atrazine + UAN did not reduce barnyardgrass biomass; in contrast tolpyralate + atrazine + MSO or MSO + UAN reduced barnyardgrass biomass $\geq 81 \%$. Metzger et al. [10] reported $>90 \%$ of control of barnyardgrass with tolpyralate + atrazine $+\mathrm{MSO}+\mathrm{UAN}\left(30\right.$ or $\left.40+1000 \mathrm{~g} \cdot \mathrm{ai} \cdot \mathrm{ha}^{-1}\right)$ at 2 and $4 \mathrm{WAA}$, however similar to results of this study, by 8 WAA control decreased slightly to $86 \%$. Previous greenhouse studies by Bunting et al. 2004 [28] showed that barnyardgrass control with foramsulfuron was greatest when MSO was the adjuvant and there was no benefit to the addition of a nitrogen source, similar to results of this study in 
Table 16. Significance of main effects and interactions for barnyardgrass (Echinochloa crus-galli (L.) P. Beauv.) control, density and biomass in corn with Roundup Weather$\mathrm{MAX}^{\circledast}$ plus tolpyralate plus atrazine from six field experiments conducted in Ontario in 2018 and 2019.

\begin{tabular}{|c|c|c|c|c|c|}
\hline \multirow{2}{*}{ Main effects } & \multicolumn{3}{|c|}{ Visible Weed Control (\%) } & \multirow{2}{*}{$\begin{array}{l}\text { Density }{ }^{\mathrm{f}} \\
\left(\text { no } \cdot \mathrm{m}^{-2}\right)\end{array}$} & \multirow{2}{*}{$\begin{array}{c}\text { Biomass } \\
\left(\mathrm{g} \cdot \mathrm{m}^{-2}\right)\end{array}$} \\
\hline & $2 \mathrm{WAA}^{\mathrm{a}}$ & $4 \mathrm{WAA}$ & $8 \mathrm{WAA}$ & & \\
\hline \multicolumn{6}{|l|}{ Roundup WeatherMAX $X^{\oplus}\left(g \cdot a e h a^{-1}\right)$} \\
\hline 0 & 49 & 47 & 50 & $34.9 \mathrm{a}$ & 29.7 \\
\hline 900 & 94 & 95 & 90 & $17.2 \mathrm{a}$ & 1.7 \\
\hline $\mathrm{SE}^{\mathrm{b}}$ & 2 & 2 & 2 & 1.9 & 1.7 \\
\hline Roundup WeatherMAX ${ }^{\oplus} \mathrm{P}$-value & $<0.0001$ & $<0.0001$ & 0.0009 & 0.0724 & 0.0033 \\
\hline \multicolumn{6}{|l|}{ Tolpyralate + atrazine treatments } \\
\hline No tank mix partner & 37 & 37 & 33 & $28.9 \mathrm{a}$ & 16.2 \\
\hline Tolpyralate + Atrazine & 66 & 70 & 71 & $34.9 \mathrm{a}$ & 20.8 \\
\hline Tolpyralate + Atrazine $+\mathrm{MSO}^{\mathrm{d}}$ & 93 & 93 & 89 & $22.9 \mathrm{a}$ & 3.9 \\
\hline Tolpyralate + Atrazine $+\mathrm{UAN}^{\mathrm{e}}$ & 70 & 69 & 73 & $25.3 \mathrm{a}$ & 20.7 \\
\hline Tolpyralate + Atrazine + MSO + UAN & 95 & 94 & 89 & $20.9 \mathrm{a}$ & 3.1 \\
\hline$S E^{b}$ & 2 & 2 & 2 & 1.9 & 1.7 \\
\hline Tolpyralate + atrazine $\mathrm{P}$-value & $<0.0001$ & $<0.0001$ & $<0.0001$ & 0.3873 & 0.0002 \\
\hline \multicolumn{6}{|l|}{ Interaction } \\
\hline $\begin{array}{c}\text { Roundup WeatherMAX }{ }^{\otimes \star} \text { tolpyralate }+ \\
\text { atrazine } \mathrm{P} \text {-value }\end{array}$ & $<0.0001$ & $<0.0001$ & $<0.0001$ & 0.3385 & 0.0002 \\
\hline
\end{tabular}

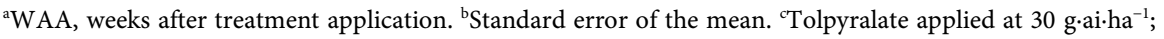
atrazine applied at $560 \mathrm{~g} \cdot \mathrm{ai} \cdot \mathrm{ha}^{-1} .{ }^{\mathrm{d}} \mathrm{MSO}, 0.50 \% \mathrm{v} / \mathrm{v}$. ${ }^{\mathrm{e}} \mathrm{UAN}, 2.50 \% \mathrm{v} / \mathrm{v}$. ${ }^{\mathrm{f}}$ Density and biomass collected 8 WAA. Means within column followed by the same lowercase letter are not statistically different according to Tukey-Kramer's LSD $(P=0.05)$.

which barnyardgrass control with MSO or MSO + UAN did not differ. In the same study, the addition of a nitrogen source had a greater effect on weed control when added to other adjuvants like a COC or a non-ionic surfactant. Roundup WeatherMAX ${ }^{\circledast}$ controlled barnyardgrass $88 \%-93 \%$ at 2,4 and 8 WAA; there was no improvement in barnyardgrass control when tolpyralate + atrazine was added to Roundup WeatherMAX ${ }^{\circledR}$. Starke and Oliver 1998 [23] reported that depending on rate, tank mixes of Roundup WeatherMAX ${ }^{\circledast}$ plus sulfentrazone, imazethapyr and chlorimuron were antagonistic for barnyardgrass control, however this was not the case with Roundup WeatherMAX ${ }^{\circledR}+$ tolpyralate + atrazine. The addition of Roundup WeatherMAX ${ }^{\oplus}$ to tolpyralate + atrazine and tolpyralate + atrazine + UAN improved barnyardgrass control at all evaluation timings and resulted in greater biomass reductions. There was no increase in barnyardgrass control when Roundup WeatherMAX ${ }^{\circledR}$ was added to tolpyralate + atrazine + MSO or MSO + UAN . 
Table 17. Barnyardgrass (Echinochloa crus-galli (L.) P. Beauv.) control 2, 4, and 8 WAA and biomass in corn with Roundup WeatherMAX ${ }^{\circledast}$ plus tolpyralate plus atrazine from six field experiments conducted in Ontario in 2018 and 2019.

\begin{tabular}{|c|c|c|c|}
\hline & \multicolumn{3}{|c|}{ Roundup WeatherMAX ${ }^{\oplus}\left(\mathrm{g} \cdot \mathrm{ae} \cdot \mathrm{ha}^{-1}\right)$} \\
\hline & 0 & 900 & SE \\
\hline \multicolumn{4}{|l|}{ Visible Weed Control 2 WAA $A^{a}(\%)$} \\
\hline No tank mix partner & $0 \mathrm{cY}$ & $93 \mathrm{aZ}$ & 7 \\
\hline Tolpyralate + Atrazine ${ }^{\mathrm{b}}$ & $33 \mathrm{bY}$ & $92 \mathrm{aZ}$ & 5 \\
\hline Tolpyralate + Atrazine $+\mathrm{MSO}^{c}$ & $91 \mathrm{aZ}$ & $95 \mathrm{aZ}$ & 1 \\
\hline Tolpyralate + Atrazine + UAN $^{\mathrm{d}}$ & $40 \mathrm{bY}$ & $92 \mathrm{aZ}$ & 4 \\
\hline Tolpyralate + Atrazine + MSO + UAN & $93 \mathrm{aZ}$ & $96 \mathrm{aZ}$ & 1 \\
\hline $\mathrm{SE}^{\mathrm{e}}$ & 3 & 0.6 & \\
\hline \multicolumn{4}{|l|}{ Visible Weed Control 4 WAA (\%) } \\
\hline No tank mix partner & $0 c \mathrm{Y}$ & $93 \mathrm{aZ}$ & 7 \\
\hline Tolpyralate + Atrazine & $34 \mathrm{bY}$ & $95 \mathrm{aZ}$ & 5 \\
\hline Tolpyralate + Atrazine + MSO & $89 \mathrm{aZ}$ & $96 a Z$ & 1 \\
\hline Tolpyralate + Atrazine + UAN & $34 \mathrm{bY}$ & $94 \mathrm{aZ}$ & 5 \\
\hline Tolpyralate + Atrazine $+\mathrm{MSO}+\mathrm{UAN}$ & $92 \mathrm{aZ}$ & $96 a Z$ & 1 \\
\hline SE & 3 & 0.4 & \\
\hline \multicolumn{4}{|l|}{ Visible Weed Control 8 WAA (\%) } \\
\hline No tank mix partner & $0 c \mathrm{Y}$ & $88 \mathrm{aZ}$ & 6 \\
\hline Tolpyralate + Atrazine & $50 \mathrm{bY}$ & $88 \mathrm{aZ}$ & 4 \\
\hline Tolpyralate + Atrazine + MSO & $84 \mathrm{aZ}$ & $93 \mathrm{aZ}$ & 2 \\
\hline Tolpyralate + Atrazine + UAN & $52 \mathrm{bY}$ & $89 \mathrm{aZ}$ & 4 \\
\hline Tolpyralate + Atrazine + MSO + UAN & $84 \mathrm{aZ}$ & $92 \mathrm{aZ}$ & 2 \\
\hline SE & 3 & 1 & \\
\hline \multicolumn{4}{|l|}{ Biomass $\left(\mathrm{g} \cdot \mathrm{m}^{-2}\right)$} \\
\hline No tank mix partner & $43.6 \mathrm{aY}$ & $1.9 \mathrm{aZ}$ & 4.1 \\
\hline Tolpyralate + Atrazine & $55.2 \mathrm{aY}$ & $2.0 \mathrm{aZ}$ & 4.5 \\
\hline Tolpyralate + Atrazine $+\mathrm{MSO}$ & $8.3 \mathrm{bZ}$ & $1.4 \mathrm{aZ}$ & 1.6 \\
\hline Tolpyralate + Atrazine + UAN & $54.6 \mathrm{aY}$ & $1.6 a Z$ & 5.6 \\
\hline Tolpyralate + Atrazine $+\mathrm{MSO}+\mathrm{UAN}$ & $5.4 \mathrm{bZ}$ & $1.5 \mathrm{aZ}$ & 1.1 \\
\hline SE & 3.2 & 0.2 & \\
\hline
\end{tabular}

${ }^{\mathrm{a}} \mathrm{WAA}$, weeks after treatment application. ${ }^{\mathrm{b}}$ Tolpyralate applied at $30 \mathrm{~g} \cdot \mathrm{ai} \cdot \mathrm{ha}^{-1}$; atrazine applied at 560 g.ai $\cdot \mathrm{ha}^{-1} .{ }^{\mathrm{c}} \mathrm{MSO}, 0.50 \% \mathrm{v} / \mathrm{v} .{ }^{\mathrm{d}} \mathrm{UAN}, 2.50 \% \mathrm{v} / \mathrm{v}$. ${ }^{\mathrm{e}}$ Standard error of the mean. Means within column followed by the same lowercase letter, or means within row followed by the same uppercase letter, are not statistically different according to Tukey-Kramer's LSD $(P=0.05)$. 


\subsection{Green Foxtail}

Green foxtail control and biomass varied with Roundup WeatherMAX ${ }^{\otimes}$ and tolpyralate + atrazine plus adjuvant treatment, producing a significant Roundup WeatherMAX ${ }^{\oplus}$ by tolpyralate plus adjuvant interaction for control at 2,4 and 8 WAA $(P<0.0001)$ and biomass $(P<0.0001)$ (Table 18). Data analysis indicated that the Roundup WeatherMAX ${ }^{\oplus}$ by tolpyralate + atrazine interaction was not significant for green foxtail density $(P=0.1407)$, however for both factors there were significant main effects. Averaged across all levels of tolpyralate + atrazine plus adjuvant treatments, the presence of Roundup WeatherMAX ${ }^{\circledR}$ reduced green foxtail density by $74 \%$ (Table 18). Across 0 and 900 g.ae $\cdot \mathrm{ha}^{-1}$ of Roundup WeatherMAX ${ }^{\oplus}$, tolpyralate + atrazine reduced green foxtail density $45 \%$, the addition of MSO + UAN to tolpyralate + atrazine decreased foxtail density a further $21 \%$.

Tolpyralate + atrazine controlled green foxtail $38 \%-42 \%$ at 2, 4 and 8 WAA (Table 19). The addition of UAN to tolpyralate + atrazine did not improve green foxtail control. In contrast, tolpyralate + atrazine + MSO or MSO + UAN controlled green foxtail $\geq 84 \%$. Tolpyralate + atrazine and tolpyralate + atrazine + UAN reduced green foxtail biomass $\leq 21 \%$. In contrast, tolpyralate + atrazine + MSO or MSO + UAN reduced green foxtail biomass $\geq 95 \%$. MSO has been reported to influence spray droplet contact angle and surface tension, allowing for greater droplet spread on the leaf surface and greater absorption in the plant [15]. Efficacy of topramezone was increased 1.5-fold with the addition of MSO to the spray mixture. Metzger et al. 2018 [27] and 2019 [10] reported excellent control of green foxtail 2 and 4 WAA with tolpyralate + atrazine $(30+1000$ g.ai.ha ${ }^{-1}$ ) plus recommended adjuvants. Roundup WeatherMAX ${ }^{\circledast}$ controlled green foxtail $\geq 88 \%$ at 2,4 and 8 WAA. There was no improvement in green foxtail control when tolpyralate + atrazine was added to Roundup Weather$\mathrm{MAX}^{\oplus}$. Roundup WeatherMAX ${ }^{\oplus}$ reduced green foxtail biomass $99 \%$; there was no further decrease in green foxtail biomass when tolpyralate + atrazine was added to Roundup WeatherMAX ${ }^{\oplus}$. The addition of Roundup WeatherMAX ${ }^{\oplus}$ to all tolpyralate + atrazine treatments improved green foxtail control at 8 WAA and resulted in further biomass reduction, however at 2 and 4 WAA, not all treatments benefited from the addition of Roundup WeatherMAX.

\subsection{Crop Injury}

Crop injury was less than $10 \%$ with all the treatments evaluated (data not presented). In contrast, Soltani et al. 2018 [20] reported increased corn injury from 2,4-D when it was co-applied with Roundup WeatherMAX ${ }^{\bullet}$. Metzger et al. 2019 [9] reported similar results to this study where tolpyralate + atrazine caused minimal corn injury.

\subsection{Crop Yield}

The Roundup WeatherMAX ${ }^{\oplus}$ by tolpyralate + atrazine interaction was significant for corn grain yield $(P<0.0001)$ (Table 20). Reduced weed interference 
Table 18. Significance of main effects and interactions for green foxtail (Setaria viridis (L.) P. Beauv.) control, density and biomass in corn with Roundup WeatherMAX ${ }^{\circledast}$ plus tolpyralate plus atrazine from six field experiments conducted in Ontario in 2018 and 2019.

\begin{tabular}{|c|c|c|c|c|c|}
\hline \multirow{2}{*}{ Main effects } & \multicolumn{3}{|c|}{ Visible Weed Control (\%) } & \multirow{2}{*}{$\begin{array}{l}\text { Density } \\
\left(\text { no } \cdot m^{-2}\right)\end{array}$} & \multirow{2}{*}{$\begin{array}{c}\text { Biomass }^{\mathrm{f}} \\
\left(\mathrm{g} \cdot \mathrm{m}^{-2}\right)\end{array}$} \\
\hline & $2 \mathrm{WAA}^{\mathrm{a}}$ & $4 \mathrm{WAA}$ & 8 WAA & & \\
\hline \multicolumn{6}{|l|}{ Roundup WeatherMAX $X^{\oplus}\left(g \cdot a e h a^{-1}\right)$} \\
\hline 0 & 51 & 49 & 47 & $56.6 \mathrm{a}$ & 72.9 \\
\hline 900 & 96 & 96 & 92 & $14.3 \mathrm{~b}$ & 1.0 \\
\hline $\mathrm{SE}^{\mathrm{b}}$ & 2 & 2 & 2 & 3.5 & 4.9 \\
\hline Roundup WeatherMAX ${ }^{\circledR}$ P-value & $<0.0001$ & $<0.0001$ & $<0.0001$ & 0.0110 & 0.0010 \\
\hline \multicolumn{6}{|l|}{ Tolpyralate + atrazine treatments } \\
\hline No tank mix partner & 40 & 38 & 33 & $73.3 \mathrm{a}$ & 83.2 \\
\hline Tolpyralate + Atrazine & 73 & 72 & 70 & $40.2 \mathrm{ab}$ & 50.9 \\
\hline Tolpyralate + Atrazine $+\mathrm{MSO}^{\mathrm{d}}$ & 94 & 94 & 90 & $29.9 \mathrm{bc}$ & 3.7 \\
\hline Tolpyralate + Atrazine + UAN ${ }^{e}$ & 73 & 72 & 71 & $41.7 \mathrm{abc}$ & 40.1 \\
\hline Tolpyralate + Atrazine + MSO + UAN & 95 & 94 & 90 & $24.7 \mathrm{c}$ & 3.4 \\
\hline $\mathrm{SE}^{\mathrm{b}}$ & 2 & 2 & 2 & 3.5 & 4.9 \\
\hline Tolpyralate + atrazine P-value & $<0.0001$ & $<0.0001$ & $<0.0001$ & 0.0005 & $<0.0001$ \\
\hline \multicolumn{6}{|l|}{ Interaction } \\
\hline $\begin{array}{l}\text { Roundup WeatherMAX }{ }^{\otimes *} \text { tolpyralate }+ \\
\text { atrazine } \mathrm{P} \text {-value }\end{array}$ & $<0.0001$ & $<0.0001$ & $<0.0001$ & 0.1407 & $<0.0001$ \\
\hline
\end{tabular}

${ }^{\mathrm{a}} \mathrm{WAA}$, weeks after treatment application. ${ }^{\mathrm{b}}$ Standard error of the mean. ${ }^{\mathrm{c}}$ Tolpyralate applied at $30 \mathrm{~g} \cdot \mathrm{ai} \cdot \mathrm{ha}{ }^{-1}$; atrazine applied at 560 g.ai $\cdot \mathrm{ha}^{-1} \cdot{ }^{\mathrm{d}} \mathrm{MSO}, 0.50 \% \mathrm{v} / \mathrm{v}$. ${ }^{\mathrm{e}} \mathrm{UAN}, 2.50 \% \mathrm{v} / \mathrm{v}$. ${ }^{\mathrm{f}}$ Density and biomass collected 8 WAA. Means within column followed by the same lowercase letter are not statistically different according to Tukey-Kramer's LSD $(P=0.05)$.

with all the herbicide treatments evaluated resulted in higher grain yield than the no tank mix partner (Table 21). Weed interference reduced corn yield up to 54\% (no tank mix partner without Roundup WeatherMAX compared to the highest yielding treatment) in this study. The reduced corn yield in the no tank mix partner without Roundup WeatherMAX ${ }^{\oplus}$ treatment is expected due to high weed interference and is similar to findings of Soltani et al. 2016 [2] that reported an average of 50\% corn grain yield loss in the absence of weed control strategies from a meta-analysis across North America. Compared to no tank mix partner, tolpyralate + atrazine resulted in an increase in corn yield of 63\%; there was no further increase in corn yield when UAN was added to tolpyralate + atrazine. The addition of MSO or MSO + UAN improved corn yield $32 \%$ compared to tolpyralate + atrazine alone. Consistent with the weed control evaluations, tolpyralate + atrazine $+\mathrm{MSO}$ or MSO + UAN provided the best weed control and resulted in grain corn yield of $\geq 12.2 \mathrm{t}^{\mathrm{h}} \mathrm{ha}^{-1}$. Roundup WeatherMAX ${ }^{\odot}$ improved corn yield by $6.4 \mathrm{t} \cdot \mathrm{ha}^{-1}$ compared to no tank mix partner. There was no further increase in corn yield with the addition of tolpyralate + atrazine to 
Table 19. Green foxtail (Setaria viridis (L.) P. Beauv.) control 2, 4, and 8 WAA and biomass in corn with Roundup WeatherMAX ${ }^{\circledR}$ plus tolpyralate plus atrazine from six field experiments conducted in Ontario in 2018 and 2019.

\begin{tabular}{|c|c|c|c|}
\hline & \multicolumn{3}{|c|}{ Roundup WeatherMAX $\left(\mathrm{g} \cdot \mathrm{ae} \cdot \mathrm{ha}^{-1}\right)$} \\
\hline & 0 & 900 & SE \\
\hline \multicolumn{4}{|l|}{ Visible Weed Control 2 WA $A^{a}(\%)$} \\
\hline No tank mix partner & $0 \mathrm{cY}$ & $96 a Z$ & 7 \\
\hline Tolpyralate + Atrazine $\mathrm{e}^{\mathrm{b}}$ & $38 \mathrm{bY}$ & $96 a Z$ & 5 \\
\hline Tolpyralate + Atrazine $+\mathrm{MSO}^{\mathrm{c}}$ & $91 \mathrm{aZ}$ & $97 a Z$ & 1 \\
\hline Tolpyralate + Atrazine $+\mathrm{UAN}^{\mathrm{d}}$ & $44 \mathrm{bY}$ & $95 \mathrm{aZ}$ & 4 \\
\hline Tolpyralate + Atrazine $+\mathrm{MSO}+\mathrm{UAN}$ & $93 a Z$ & $97 \mathrm{aZ}$ & 1 \\
\hline $\mathrm{SE}^{\mathrm{e}}$ & 3 & 0.4 & \\
\hline \multicolumn{4}{|l|}{ Visible Weed Control4 WAA (\%) } \\
\hline No tank mix partner & $0 \mathrm{cY}$ & $94 \mathrm{aZ}$ & 7 \\
\hline Tolpyralate + Atrazine & $39 \mathrm{bY}$ & $95 \mathrm{aZ}$ & 5 \\
\hline Tolpyralate + Atrazine $+\mathrm{MSO}$ & $90 \mathrm{aY}$ & $97 \mathrm{aZ}$ & 1 \\
\hline Tolpyralate + Atrazine + UAN & $38 \mathrm{bY}$ & $96 a Z$ & 5 \\
\hline Tolpyralate + Atrazine $+\mathrm{MSO}+\mathrm{UAN}$ & $92 \mathrm{aZ}$ & $96 a Z$ & 1 \\
\hline SE & 3 & 0.4 & \\
\hline \multicolumn{4}{|l|}{ Visible Weed Control 8 WAA (\%) } \\
\hline No tank mix partner & $0 \mathrm{cY}$ & $88 \mathrm{aZ}$ & 6 \\
\hline Tolpyralate + Atrazine & $42 \mathrm{bY}$ & $91 \mathrm{aZ}$ & 4 \\
\hline Tolpyralate + Atrazine $+\mathrm{MSO}$ & $86 \mathrm{aY}$ & $94 \mathrm{aZ}$ & 1 \\
\hline Tolpyralate + Atrazine + UAN & $44 \mathrm{bY}$ & $92 \mathrm{aZ}$ & 4 \\
\hline Tolpyralate + Atrazine $+\mathrm{MSO}+\mathrm{UAN}$ & $84 \mathrm{aY}$ & $94 \mathrm{aZ}$ & 1 \\
\hline SE & 3 & 1 & \\
\hline \multicolumn{4}{|l|}{ Biomass $\left(\mathrm{g} \cdot \mathrm{m}^{-2}\right)$} \\
\hline No tank mix partner & $148.7 \mathrm{aY}$ & $1.9 \mathrm{aZ}$ & 16.2 \\
\hline Tolpyralate + Atrazine & $125.2 \mathrm{aY}$ & $0.9 \mathrm{aZ}$ & 12.8 \\
\hline Tolpyralate + Atrazine + MSO & $8.0 \mathrm{bY}$ & $0.7 \mathrm{aZ}$ & 0.8 \\
\hline Tolpyralate + Atrazine + UAN & $117.2 \mathrm{aY}$ & $1.1 \mathrm{aZ}$ & 11.0 \\
\hline Tolpyralate + Atrazine $+\mathrm{MSO}+\mathrm{UAN}$ & $7.3 \mathrm{bY}$ & $0.7 \mathrm{aZ}$ & 0.8 \\
\hline SE & 9 & 0.2 & \\
\hline
\end{tabular}

${ }^{a}$ WAA, weeks after treatment application. ${ }^{\mathrm{b}}$ Tolpyralate applied at $30 \mathrm{~g} \cdot \mathrm{ai} \cdot \mathrm{ha}^{-1}$; atrazine applied at 560 g.ai.ha ${ }^{-1}$. ${ }^{\mathrm{c}} \mathrm{MSO}, 0.50 \% \mathrm{v} / \mathrm{v}$. ${ }^{\mathrm{d}} \mathrm{UAN}, 2.50 \% \mathrm{v} / \mathrm{v}$. ${ }^{\mathrm{e} S t a n d a r d}$ error of the mean. Means within column followed by the same lowercase letter, or means within row followed by the same uppercase letter, are not statistically different according to Tukey-Kramer's LSD $(P=0.05)$. 
Table 20. Significance of main effects and interactions for grain corn yield with Roundup WeatherMAX ${ }^{\circledast}$ plus tolpyralate plus atrazine from five field experiments conducted near Ridgetown and Exeter, Ontario in 2018 and 2019.

\begin{tabular}{|c|c|}
\hline Main effects & Yield $\left(\mathrm{t} \cdot \mathrm{ha}^{-1}\right)$ \\
\hline \multicolumn{2}{|l|}{ Roundup WeatherMAX $X^{\oplus}\left(g \cdot a e \cdot h a^{-1}\right)$} \\
\hline 0 & 9.8 \\
\hline 900 & 12.0 \\
\hline $\mathrm{SE}^{\mathrm{b}}$ & 0.2 \\
\hline Roundup WeatherMAX ${ }^{\oplus} \mathrm{P}$-value & 0.0574 \\
\hline \multicolumn{2}{|l|}{ Tolpyralate + atrazine treatments ${ }^{\mathcal{c}}$} \\
\hline No tank mix partner & 8.9 \\
\hline Tolpyralate + Atrazine & 10.7 \\
\hline Tolpyralate + Atrazine $+\mathrm{MSO}^{\mathrm{d}}$ & 12.4 \\
\hline Tolpyralate + Atrazine + UAN $^{e}$ & 10.5 \\
\hline Tolpyralate + Atrazine $+\mathrm{MSO}+\mathrm{UAN}$ & 12.1 \\
\hline $\mathrm{SE}^{\mathrm{b}}$ & 0.2 \\
\hline Tolpyralate + atrazine P-value & $<0.0001$ \\
\hline \multicolumn{2}{|l|}{ Interaction } \\
\hline Roundup WeatherMAX ${ }^{\oplus \star}$ tolpyralate + atrazine P-value & $<0.0001$ \\
\hline
\end{tabular}

Table 21. Corn yields achieved with Roundup WeatherMAX ${ }^{\circledR}$ plus tolpyralate plus atrazine from five field experiments conducted in Ontario in 2018 and 2019.

\begin{tabular}{|c|c|c|c|}
\hline & \multicolumn{3}{|c|}{ Roundup WeatherMAX ${ }^{\oplus}$ Rate (g.ae $\cdot \mathrm{ha}^{-1}$ ) } \\
\hline & 0 & 900 & SE \\
\hline $\operatorname{Yield}\left(\mathrm{t} \cdot \mathrm{ha}^{-1}\right)$ & \multicolumn{3}{|c|}{ - } \\
\hline No tank mix partner & $5.7 \mathrm{cY}$ & $12.1 \mathrm{aZ}$ & 0.6 \\
\hline Tolpyralate + Atrazine $^{\mathrm{b}}$ & $9.3 \mathrm{bY}$ & $11.9 \mathrm{aZ}$ & 0.5 \\
\hline Tolpyralate + Atrazine $+\mathrm{MSO}^{\mathrm{c}}$ & $12.3 \mathrm{aZ}$ & $12.5 \mathrm{aZ}$ & 0.2 \\
\hline Tolpyralate + Atrazine $+\mathrm{UAN}^{\mathrm{d}}$ & $9.4 \mathrm{bZ}$ & $11.5 \mathrm{aZ}$ & 0.4 \\
\hline Tolpyralate + Atrazine $+\mathrm{MSO}+\mathrm{UAN}$ & $12.2 \mathrm{aZ}$ & $12.1 \mathrm{aZ}$ & 0.2 \\
\hline $\mathrm{SE}^{\mathrm{a}}$ & 0.3 & 0.1 & \\
\hline
\end{tabular}

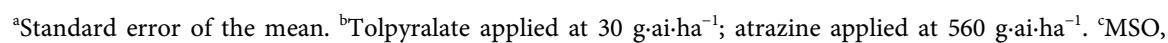
$0.50 \% \mathrm{v} / \mathrm{v}$. ${ }^{\mathrm{d}} \mathrm{UAN}, 2.50 \% \mathrm{v} / \mathrm{v}$. Means within column followed by the same lowercase letter, or means within row followed by the same uppercase letter, are not statistically different according to Tukey-Kramer's LSD $(P=0.05)$.

Roundup WeatherMAX ${ }^{\circledast}$ compared to Roundup WeatherMAX ${ }^{\circledast}$ alone. Research by Metzger et al. 2019 [10] found that tolpyralate + atrazine $(30+1000)$ applied early or mid-POST resulted in corn yields that were comparable to the weed free 
control, however when applications were delayed to late-POST corn yield was reduced. The addition of Roundup WeatherMAX ${ }^{\odot}$ to tolpyralate + atrazine, in the absence of adjuvants, increased grain corn yield $27 \%$.

\section{Conclusions}

Results from this study indicate that the addition of MSO to tolpyralate + atrazine improved the control of velvetleaf, pigweed, common ragweed, lambsquarters, ladysthumb, barnyardgrass and green foxtail with the exception of flower-of-an-hour at $2 \mathrm{WAA}$, common ragweed at $8 \mathrm{WAA}$ and wild mustard and ladysthumb at all evaluations. The addition of UAN to tolpyralate + atrazine did not improve the control of velvetleaf, pigweed, common ragweed, lambsquarters, ladysthumb, wild mustard, flower-of-an-hour, barnyardgrass and green foxtail. Generally, there was no benefit of adding UAN to tolpyralate + atrazine $+\mathrm{MSO}$ in respect to weed control or corn yield. The current tolpyralate label recommends the use of both MSO and UAN for optimal weed control. The addition of MSO and UAN to tolpyralate + atrazine would require the farmer to spend an additional $\$ 10.00 \mathrm{ha}^{-1}$ (Agris Co-operative Ltd., personal communication). Based on this research, it is not necessary to add UAN to the spray tank, potentially allowing farmers to save up to $\$ 2.50 \mathrm{ha}^{-1}$; this cost may appear small but may be substantial over many hectares.

Roundup WeatherMAX ${ }^{\circledR}$ controlled velvetleaf (96\%), pigweed (97\%), common ragweed (88\%), lambsquarters (91\%), ladysthumb (94\%), wild mustard (97\%), flower-of-an-hour (84\%), barnyard grass (93\%) and green foxtail (96\%) at 2 WAA. There was no benefit of adding tolpyralate + atrazine to Roundup WeatherMAX ${ }^{\oplus}$ in respect to weed control with exception of lambsquarters control at 2 WAA and reduction in lambsquarters density at 8 WAA.

Although accentuated crop injury was not observed in this study with the addition of Roundup WeatherMAX ${ }^{\otimes}$ to tolpyralate + atrazine, there was an influence on weed control. The addition of Roundup WeatherMAX ${ }^{\infty}$ to tolpyralate + atrazine improved the control of velvetleaf, pigweed, common ragweed, lambsquarters, ladysthumb, wild mustard, flower-of-an-hour, barnyard grass and green foxtail; however, there was no increase in weed control when Roundup WeatherMAX ${ }^{\circledast}$ was added to tolpyralate + atrazine + MSO with the exception of flower-of-an-hour at 2 WAA.

There was an increase in corn grain yield when Roundup WeatherMAX ${ }^{\bullet}$ was added to tolpyralate + atrazine, but there was no increase in yield when Roundup WeatherMAX ${ }^{\oplus}$ was added to tolpyralate + atrazine + MSO. Over $96 \%$ of corn acres in Eastern Canada are seeded to Roundup Ready hybrids and the vast majority of those acres will have glyphosate applied POST, so the most common use of tolpyralate + atrazine will be in a tank mix with glyphosate. Based on this research, there is no need to add additional adjuvants to the tank when tolpyralate + atrazine is co-applied with Roundup WeatherMAX ${ }^{\oplus}$, potentially allowing farmers to save up to $\$ 10.00 \mathrm{ha}^{-1}$ on adjuvants. The use of multiple effective modes of action is a good resistance management practice to preserve the 
long-term effectiveness of herbicides.

This study concludes that in the absence of Roundup WeatherMAX ${ }^{\oplus}$, optimal weed control is achieved with tolpyralate + atrazine + MSO. When tolpyralate + atrazine is co-applied with Roundup WeatherMAX ${ }^{\oplus}$, no additional adjuvants are required.

\section{Acknowledgements}

Funding for this project was provided in part by ISK Biosciences Inc. and Grain Farmers of Ontario (GFO).

\section{Conflicts of Interest}

The authors declare no conflicts of interest regarding the publication of this paper.

\section{References}

[1] Page, E.R., Cerrudo, D., Westra, P., Loux, M., Smith, K., Foresman, C., Wright, H. and Swanton, C.J. (2012) Why Early Season Weed Control Is Important in Maize. Weed Science, 60, 423-430. https://doi.org/10.1614/WS-D-11-00183.1

[2] Soltani, N., Dille, J.A., Burke, I.C., Everman, W.J., VanGessel, M.J., Davis, V.M. and Sikkema, P.H. (2017) Perspectives on Potential Soybean Yield Losses from Weeds in North America. Weed Technology, 30, 148-154. https://doi.org/10.1017/wet.2016.2

[3] Swanton, C.J. and Weise, S.F. (1991) Integrated Weed Management: The Rationale and Approach. Weed Technology, 5, 657-663. https://doi.org/10.1017/S0890037X00027512

[4] Hall, M.R., Swanton, C.J. and Anderson, G.W. (1992) The Critical Period of Weed Control in Grain Corn (Zea mays). Weed Science, 40, 441-447. https://doi.org/10.1017/S0043174500051882

[5] Ahrens, H., Lange, G., Müller, T., Rosinger, C., Willms, L. and van Almsick, A. (2013) 4-Hydroxyphenylpyruvate Dioxygenase Inhibitors in Combination with Safeners: Solutions for Modern and Sustainable Agriculture. Angewandte Chemie International Edition, 52, 9388-9398. https://doi.org/10.1002/anie.201302365

[6] Hawkes, T.R. (2012) Hydroxyphenylpyruvate Dioxygenase (HPPD): The Herbicide Target. In: Krämer, W., Schirmer, U., Jeschke, P. and Witschel, M., Eds., Modern Crop Protection Compounds, 2nd Edition, Volume 1, Wiley-VCH Verlag GmbH \& Co., Weinheim, Germany.

[7] Anonymous (2019) SHIELDEX ${ }^{\oplus}$ 400SC Herbicide Label. ISK Biosciences Corporation, Concord, $\mathrm{OH}$.

[8] Metzger, B.A., Soltani, N., Raeder, A.J., Hooker, D.C., Robinson, D.E. and Sikkema, P.H. (2018) Tolpyralate Efficacy: Part 1. Biologically Effective Dose of Tolpyralate for Control of Annual Grass and Broadleaf Weeds in Corn. Weed Technology, 32, 698-706. https://doi.org/10.1017/wet.2018.82

[9] Metzger, B.A., Soltani, N., Raeder, A.J., Hooker, D.C., Robinson, D.E. and Sikkema, P.H. (2019) Effect of Hybrid Varieties, Application Timing, and Herbicide Rate on Field Corn Tolerance to Tolpyralate plus Atrazine. Weed Science, 67, 475-484. https://doi.org/10.1017/wsc.2019.34

[10] Metzger, B.A., Soltani, N., Raeder, A.J., Hooker, D.C., Robinson, D.E. and Sikkema, 
P.H. (2019) Influence of Application Timing and Herbicide Rate on the Efficacy of Tolpyralate plus Atrazine. Weed Technology, 33, 448-458. https://doi.org/10.1017/wet.2019.25

[11] Anonymous (2019) Roundup WeatherMAX ${ }^{\circledast}$ with Transorb 2 Technology. Monsanto Canada ULC, Winnipeg, Manitoba, Canada.

[12] Weed Science Society of America [WSSA] (2019) WSSA Glossary. http://wssa.net/wssa/wssa-glossary/

[13] McMullan, P.M. (2000) Utility Adjuvants. Weed Technology, 14, 792-797. https://doi.org/10.1614/0890-037X(2000)014[0792:UA]2.0.CO;2

[14] Xu, L.Y., Zhu, H.P., Ozkan, H.E., Bagley, W.E., Derksen, R.C. and Krause, C.R. (2010) Adjuvant Effects on Evaporation Time and Wetted Area of Droplets on Waxy Leaves. American Society of Agricultural and Biological Engineers, 51, 13-20. https://doi.org/10.13031/2013.29495

[15] Zhang, J., Jaeck, O., Menegat, A., Zhang, Z., Gerhards, R. and Ni, H. (2013) The Mechanism of Methylated Seed Oil on Enhancing Biological Efficacy of Topramezone on Weeds. PLoS ONE, 8, e74280. https://doi.org/10.1371/journal.pone.0074280

[16] Gronwald, J.W., Jourdan, S.W., Wyse, D.L., Somers, D.A. and Magnusson, M.U. (1993) Effect of Ammonium Sulfate on Absorption of Imazethapyr by Quackgrass (Elytrigia repens) and Maize (Zea mays) Cell Suspension Cultures. Weed Science, 41, 325-334. https://doi.org/10.1017/S0043174500052012

[17] Woznica, Z., Nalewaja, J.D., Messersmith, C.G. and Milkowski, P. (2003) Quinclorac Efficacy as Affected by Adjuvants and Spray Carrier Water. Weed Technology, 17, 582-588.

https://doi.org/10.1614/0890-037X(2003)017[0582:QEAABA]2.0.CO;2

[18] Idziak, R., Skrzypczak, W., Waligora, H. and Woznica, Z. (2013) The Effect of Mesotrione Applied with Adjuvants on Weed Control Efficacy and Forage Sorghum Tolerance. Turkish Journal of Agriculture and Forestry, 37, 265-270.

[19] Bayer (2019) Roundup. https://www.roundup.ca/en/the-roundup-advantage

[20] Soltani, N., Shropshire, C. and Sikkema, P.H. (2018) Tank Mixture of Roundup WeatherMAX ${ }^{\bowtie}$ with 2, 4-D Accentuates 2, 4-D Injury in Roundup WeatherMAX -Resistant Corn. Canadian Journal of Plant Science, 98, 889-896. https://doi.org/10.1139/cjps-2017-0241

[21] Deen, W., Hamill, A., Shropshire, C., Soltani, N. and Sikkema, P.H. (2006) Control of Volunteer Glyphosate-Resistant Corn (Zea mays) in Glyphosate-Resistant Soybean (Glycine max). Weed Technology, 20, 261-266. https://doi.org/10.1614/WT-02-128.1

[22] Eubank, T.W., Nandula, V.K., Reddy, K.N., Poston, D.H. and Shaw, D.R. (2013) Saflufenacil Efficacy on Horseweed and Its Interaction with Glyphosate. Weed Biology and Management, 13, 135-143. https://doi.org/10.1111/wbm.12022

[23] Starke, R.J. and Oliver, L.R. (1998) Interaction of Glyphosate with Chlorimuron, Fomesafen, Imazethapyr, and Sulfentrazone. Weed Science, 46, 652-660. https://doi.org/10.1017/S0043174500089670

[24] Bilyea, D. (2016) Ontario Weed Survey. University of Guelph Ridgetown Campus, Ridgetown.

[25] Hatterman-Valenti, H., Pitty, A. and Owen, M. (2011) Environmental Effects on Velvetleaf (Abutilon theophrasti) Epicuticular Wax Deposition and Herbicide Absorption. Weed Science, 59, 14-21. https://doi.org/10.1614/WS-D-10-00061.1 
[26] Hinz, J.R. and Owen, M.D.K. (1994) Effect of Drought Stress on Velvetleaf (AbutiIon theophrasti) and Bentazon Efficacy. Weed Science, 42, 76-81. https://doi.org/10.1017/S0043174500084198

[27] Metzger, B.A., Soltani, N., Raeder, A.J., Hooker, D.C., Robinson, D.E. and Sikkema, P.H. (2018) Tolpyralate Efficacy: Part 2. Comparison of Three Group 27 Herbicides Applied POST for Annual Grass and Broadleaf Weed Control in Corn. Weed Technology, 32, 707-713. https://doi.org/10.1017/wet.2018.81

[28] Bunting, J.A., Spraque, C.L. and Reichers, D.E. (2004) Absorption and Activity of Foramsulfuron in Giant Foxtail (Setaria faberi) and Woolly Cupgrass (Eriochloa villosa) with Various Adjuvants. Weed Science, 52, 513-517.

https://doi.org/10.1614/WS-03-135R

[29] Dayan, F.E., Green, H.M., Weete, J.D. and Hancock, H.G. (1996) Postemergence Activity of Sulfentrazone: Effects of Surfactants and Leaf Surfaces. Weed Science, 44, 797-803. https://doi.org/10.1017/S0043174500094741

[30] Young, B.G. and Hart, S.E. (1998) Optimizing Foliar Activity of Isoxaflutole on Giant Foxtail (Setaria faberi) with Various Adjuvants. Weed Science, 46, 397-402. https://doi.org/10.1017/S0043174500090792

[31] Hutchinson, P.J., Eberlein, C.V. and Tonks, D.J. (2004) Broadleaf Weed Control and Potato Crop Safety with Postemergence Rimsulfuron, Metribuzin, and Adjuvant Combinations. Weed Technology, 18, 750-756.

https://doi.org/10.1614/WT-03-172R1 